\title{
Analysis of Wake Structures Behind an Oscillating Square Cylinder Using Dynamic Mode Decomposition
}

\author{
Bin Wang* \\ Department of Mathematics, University of Kansas, Lawrence, KS 66045 \\ and \\ Meilin $\mathrm{Yu}^{\dagger}$ \\ Department of Mechanical Engineering, University of Maryland, Baltimore County, Baltimore, MD 21250
}

\begin{abstract}
A dynamic mode decomposition (DMD) algorithm has been used to analyze the vortex structures shedding from an oscillating square cylinder. The flow fields are simulated using a high-order accurate flux reconstruction/correction procedure via reconstruction (FR/CPR) method. The dominant dynamic modes from the wake vortex structures are then extracted from the DMD analysis. Effects of the Strouhal number and Reynolds number on the dynamic modes are studied. Results from DMD are also compared with those from proper orthogonal decomposition (POD). In this study, the capability of the two flow analysis techniques, namely DMD and POD, on capturing dominant vortex structures have been successfully demonstrated. Insights are gained into the flow physics induced by flow structure interaction.
\end{abstract}

\section{Nomenclature}

A Propagator matrix

$A_{o} \quad$ Oscillation amplitude, $A_{o}=0.2 D$

$c_{i} \quad$ The amplitude of the $i$-th dmd mode

$C$ Temporal correlation matrix of the snapshots

$d_{k}\left(t_{i}\right)$ The amplitude of the $k$-th pod mode at time instant $t_{i}$

$D \quad$ Side length of the square cylinder

$f \quad$ Oscillation frequency of the cylinder

Re Reynolds number, $R e=\frac{\rho U_{\infty} D}{\mu}$

St Strouhal number, $S t=\frac{2 f A_{o}^{\mu}}{U_{\infty}}$

$U_{\infty}$ free stream velocity

$\lambda_{i} \quad$ The growth rate of the $i$-th dmd mode

$\mu \quad$ Dynamic viscosity of the fluid

$\rho \quad$ Fluid density

\footnotetext{
${ }^{*}$ Graduate Student, Department of Mathematics, University of Kansas. Email: b_wang@ku.edu

${ }^{\dagger}$ Assistant Professor, Department of Mechanical Engineering, University of Maryland, Baltimore County, AIAA senior member. Email: mlyu@umbc.edu
} 
$\phi_{i} \quad$ The $i$-th dmd mode

$\psi_{k} \quad$ The $k$-th pod mode

$\omega_{i} \quad$ The frequency of the $i$-th dmd mode

\section{Introduction}

Flow past bluff body has been extensively studied due to its practical importance to engineering and scientific merit to fluid mechanics. Vortex shedding behind a bluff body can induce vibration of structures, which can be detrimental to structural safety. Knowledge about flow-induced unsteady loading on structures is vital for aero-hydrodynamic design of these structures and flow control over them. In particular, rectangular cylinders resemble a wide range of constructed facilities such as bridges, buildings, and offshore structures. Despite its importance, flow past these geometries is less explored compared with its circular counterpart. In this study, we focus on flow past square cylinders undergoing forced harmonic oscillation.

Many numerical ${ }^{1-3}$ and experimental ${ }^{4}$ studies in flow past oscillating square cylinder are primarily concerned with characteristics of vortex shedding, vortex structures, aerodynamic forces, and 'lock-on' state. 'Lock-on' refers to the phenomenon that when a bluff body is forced to oscillate at a frequency close to its natural vortex shedding frequency, vortex shedding from the body will synchronize with the forced oscillation frequency. This can occur during certain oscillation frequency band. The characteristic flow features associated with this synchronization band not only are important to the structural dynamics but also exhibit interesting flow physics. Note that the vortex shedding characteristics can change with different Reynolds numbers. In this paper, we will study flow structures behind a square cylinder undergoing forced oscillation in the vicinity of lock-on state at low Reynolds numbers.

Recent advances in scale-resolving numerical simulation techniques and computing power have enabled high-fidelity simulation of vortex-dominated flows. Flux reconstruction/correction procedure via reconstruction $(\mathrm{FR} / \mathrm{CPR})^{5-7}$ is a recently developed finite-difference like discontinuous high-order method for unstructured grids. Several comprehensive reviews of recent development of the FR/CPR method can be found in Refs. [8,9]. In this work, we use the high-order FR/CPR method developed for incompressible flow to simulate the flow past the oscillating cylinder at low Reynolds numbers. ${ }^{11,12}$ In the flow solver, an arbitrary Lagrangian-Eulerian (ALE) method with implicit geometric conservation law (GCL) treatment is used to handle the moving/deformable grid in incompressible flow simulation. This solver has been verified and validated with series of numerical and experimental benchmarks.

One tradeoff with high-resolution simulation is the difficulty with exacting critical flow features due to the large data set. Popular data processing techniques that can be used to circumvent this barrier include, but not limited to, dynamic mode decomposition(DMD) and proper orthogonal decomposition (POD). DMD is a recently proposed data-based mode decomposition technique. ${ }^{14,15}$ Different from the POD method which ranks the flow structures by energy, DMD captures dominant dynamic behaviors encoded in the data sequence by frequency. So far this method and its variations have been successfully applied to a range of fluid flow problems. ${ }^{15-22}$ One objective of this paper is to extract dominant dynamical features of flow past forced oscillating square cylinders from highfidelity numerical simulations results using the DMD technique. A comparison of DMD modes and POD modes is also performed to characterize special features of DMD and POD.

The rest of the paper is organized as follows. The problem description is given in Sec. II. A description of numerical methods is provided in Sec. III. The dynamic mode decomposition and proper orthogonal decomposition technique are briefly introduced in Sec. IV and Sec.V, respectively. Results and analysis are given in Sec. VI. Finally, a conclusion is drawn in Sec. VII. 


\section{Problem Description}

Flow features caused by harmonic oscillation of a square cylinder placed in the free stream are numerically studied. The square cylinder oscillates in the traverse direction of the flow with a fixed amplitude $A_{o}=0.2 D$ at a range of frequencies, where $D$ is the side length of the square cylinder. A schematic diagram of the free-stream flow past an oscillating square cylinder is shown in Figure 1. The Reynolds number $\left(R e=\frac{\rho U_{\infty} D}{\mu}\right)$, based on the width of the cylinder and the upstream velocity, is selected as 100 and 400 . The Strouhal number, defined as $S t=\frac{2 f A_{o}}{U_{\infty}}$, varies in the range of $[0.05,0.2]$.

The computational domain is of dimensions $[-100 D, 100 D] \times[-100 D, 100 D]$ in the steam- and cross- directions respectively. An unstructured grid with a total number of 3120 quadrilateral elements is used in the simulation. The mesh is refined near the wall boundaries of the square cylinder and the wake region to capture boundary layers and wake structures, respectively. A high order scheme up to the 6 -th order is used in the simulation. More details about the numeral algorithm used is given in Sec. III.

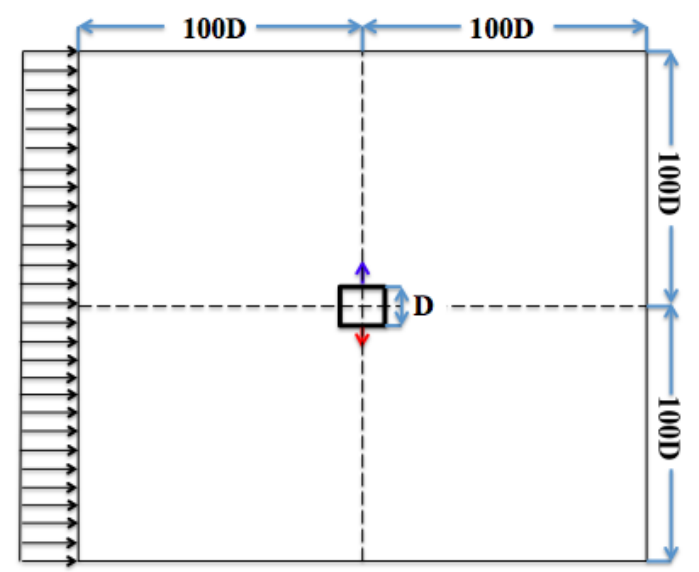

Figure 1. Schematic of the free stream flow past an oscillating square cylinder.

\section{Numerical Methods}

\section{III.A. Governing Equations}

The governing equations for unsteady incompressible viscous flow are Navier-Stokes equations, which can be written in the conservation form as:

$$
A_{t} \frac{\partial Q}{\partial t}+\nabla \cdot \mathbf{F}=0
$$

Herein, $Q=(p, u, v)$ are the primitive variables; $p$ is the pressure; $u$ and $v$ are flow velocities in the $x$ and $y$ directions; and $\mathbf{F}$ are the flux vectors, which consist of inviscid and viscous parts, i.e., $\mathbf{F}^{i n v}=\left(f^{i n v}, g^{i n v}\right)$ and $\mathbf{F}^{v i s}=\left(f^{v i s}, g^{v i s}\right)$. For two-dimensional (2D) flow, these fluxes are written as follows:

$$
f^{i n v}=\left[\begin{array}{c}
u \\
u^{2}+\frac{p}{\rho_{i n c}} \\
u v
\end{array}\right], \quad g^{i n v}=\left[\begin{array}{c}
v \\
u v \\
v^{2}+\frac{p}{\rho_{i n c}}
\end{array}\right], \quad f^{v i s}=\left[\begin{array}{c}
0 \\
\frac{\mu}{\rho_{i n c}} \frac{\partial u}{\partial x} \\
\frac{\mu}{\rho_{i n c}} \frac{\partial v}{\partial x}
\end{array}\right], \quad g^{v i s}=\left[\begin{array}{c}
0 \\
\frac{\mu}{\rho_{i n c}} \frac{\partial u}{\partial y} \\
\frac{\mu}{\rho_{i n c}} \frac{\partial v}{\partial y}
\end{array}\right]
$$


where $\rho_{\text {inc }}$ is the fluid density, and $\mu$ is the dynamic viscosity. The matrix $A_{t}$ takes the following form:

$$
A_{t}=\left[\begin{array}{lll}
0 & 0 & 0 \\
0 & 1 & 0 \\
0 & 0 & 1
\end{array}\right]
$$

To effectively solve the governing equations using modern CFD techniques developed for compressible flows, the artificial compressibility effect is introduced into Eq. (1) by augmenting the system with a pseudo-time derivative term. The new governing equations are then written as:

$$
A_{p} \frac{\partial Q}{\partial \tau}+A_{t} \frac{\partial Q}{\partial t}+\nabla \cdot \mathbf{F}=0
$$

where $A_{p}$ takes the following form:

$$
A_{p}=\left[\begin{array}{ccc}
1 / \beta & 0 & 0 \\
0 & 1 & 0 \\
0 & 0 & 1
\end{array}\right]
$$

In Eq. (5), $\beta$ is the artificial compressibility factor. It is observed that the pseudo-time derivative term $\partial Q / \partial \tau$ does not affect flow physics as long as $Q$ does not vary with respect to the pseudo-time $\tau$ at each physical time $t$. Instead, this term introduces finite response time for pressure disturbances into the incompressible flow system. This strategy provides flexibility for the numerical algorithm design of the governing equations.

To facilitate numerical discretization, Eq. (4) can be recast as

$$
\frac{\partial Q}{\partial \tau}+\nabla \cdot \mathbf{F}^{\mathbf{r}}=S
$$

where $S=-A_{t} \partial Q / \partial t$ is the source term, $\mathbf{F}^{\mathbf{r}}$ is the modified flux vector with artificial compressibility. The only difference between $\mathbf{F}^{\mathbf{r}}$ and $\mathbf{F}$ is that the inviscid fluxes $u$ and $v$ in $\mathbf{F}$ are substituted with $\beta u$ and $\beta v$ in $\mathbf{F}^{\mathbf{r}}$ respectively. For conciseness, we drop the superscript ' $\mathbf{r}$ ' in $\mathbf{F}^{\mathbf{r}}$ for the rest of the paper when no confusion arises.

\section{III.B. Spatial Discretization}

The FR/CPR formulation ${ }^{5-7}$ is used to discretize Eq. (6). Suppose that the governing equations are defined on $\Omega \times[0, T] \times[0, \Gamma]$. Herein, $\Omega$ is the spatial domain with boundary $\partial \Omega,[0, T]$ is the physical time domain, and $[0, \Gamma]$ is the pseudo-time domain. The spatial domain $\Omega$ is divided into $N$ non-overlapping elements $\Omega_{j}, j=1, \cdots, N$. The exact solution of Eq. (6) is then approximated using an element-wise continuous polynomial $Q_{h}(\mathbf{x}, t)$ defined on each element $\Omega_{j}$. By applying the weighted residual operations on each element $\Omega_{j},{ }^{23}$ the final form for the FR/CPR formulation is expressed as

$$
\frac{\partial Q}{\partial \tau}+\mathbb{P}(\nabla \cdot \mathbf{F})+\delta^{c}=S
$$

Herein, $\mathbb{P}(\nabla \cdot \mathbf{F})$ is a projection of $\nabla \cdot \mathbf{F}$ onto the polynomial space on the element $\Omega_{j}$, and $\delta^{c}$ is a correction field, which satisfies

$$
\int_{\Omega_{j}} \delta^{c} W d V=\int_{\partial \Omega_{j}}\left(F_{c o m}^{n}-F^{n}\right) W d S .
$$

In Eq. (8), $F^{n}=\mathbf{F} \cdot \mathbf{n}$ is the local normal flux on the element boundary. To ensure conservation, we also define the common flux $F_{c o m}^{n}\left(Q_{h}^{j}, Q_{h}^{j+}, \nabla Q_{h}^{j}, \nabla Q_{h}^{j+}, \mathbf{n}\right)$, where $Q_{h}^{j+}$ denotes the solution outside 
the current element $\Omega_{j}$, and $\mathbf{n}$ is the outward unit normal of $\partial \Omega_{j}$. For the inviscid common flux calculation, various approximate Riemann solvers can be used, e.g., the Roe Riemann solver. ${ }^{24}$ For the viscous common flux calculation, there also exist several choices, e.g., the second approach of Bassi and Rebay (BR2). ${ }^{25} \mathrm{~W}$ is the test function in the weighted residual operations.

\section{III.C. Dual Time Stepping Procedures}

Let $R=-\left(\mathbb{P}(\nabla \cdot \mathbf{F})+\delta^{c}\right)$, then Eq. (7) can be written as $\partial Q / \partial \tau=S+R$. A general practice is to directly solve this equation with the Newton's method. ${ }^{26}$ In this approach, the nonlinear system is firstly linearized with respect to the current solution state $Q_{h}^{m+1, n}$, where $m$ and $n$ stand for the time level in the physical and pseudo time domain, respectively. The resulting linear system becomes

$$
\left(\frac{\partial Q}{\partial \tau}\right)^{m+1, n+1}-\left(\frac{\partial R}{\partial Q}\right)^{m+1, n} \Delta Q=R^{m+1, n}+S^{m+1, n+1},
$$

where $\Delta Q=Q_{h}^{m+1, n+1}-Q_{h}^{m+1, n}$. In the physical time domain, the second-order backward differentiation formulation (BDF2) is used to discretize the term $\partial Q / \partial t$. In the pseudo-time domain, the backward Euler is used to discretize the term $\partial Q / \partial \tau$. As a result, Eq. (9) can be written as

$$
\frac{I}{\Delta \tau} \Delta Q-\left(\frac{\partial R}{\partial Q}\right)^{m+1, n} \Delta Q=R^{m+1, n}-A_{t} \frac{3 Q_{h}^{m+1, n+1}-4 Q_{h}^{m}+Q_{h}^{m-1}}{2 \Delta t}
$$

After reformulation, Eq. (10) reads

$$
\left(\frac{I}{\Delta \tau}+\frac{3 A_{t}}{2 \Delta t}-\left(\frac{\partial R}{\partial Q}\right)^{m+1, n}\right) \Delta Q=R^{m+1, n}+S^{m+1, n} .
$$

In this study, we use the block Lower-Upper Symmetric-Gauss-Seidel (LU-SGS) method to solve the linear system such derived. More details can be found in Refs. [12,13].

\section{Dynamic Mode Decomposition}

Dynamic mode decomposition is a data-based approach that can extract dynamic modes from the time-resolved datasets. This method can be applied to both data from numerical simulations and from experiments, and it does not require knowledge about governing equations of the dynamical system studied. Over the past few years, this technique has been used to analyze a variety of fluid flow problems. ${ }^{15-22}$ We give a brief overview of this method in what follows. For details about this technique, please refer to a series of work on the development of DMD. ${ }^{14-17}$

We consider the data matrix in the space-time domain with uniform temporal sampling as shown in Figure 2. Different colors distinguish different time instants from one another. The $i$-th column corresponds to the flow field snapshot at the $i$-th time instant, as denoted by $v_{i}$, for $i=1,2, \ldots, n$. For $2 \mathrm{D}$ or $3 \mathrm{D}$ measurement or simulation, we vectorize each snapshot as a column vector. Note that the data columns are equally spaced in time, which means that they are assumed to be sampled at constant time intervals $\Delta t$. We further assume a constant matrix $A$ propagates the flow field from one time instant to the consecutive time instant over the whole interval $[\Delta t, n \Delta t]$, namely,

$$
A v_{i}=v_{i+1}
$$




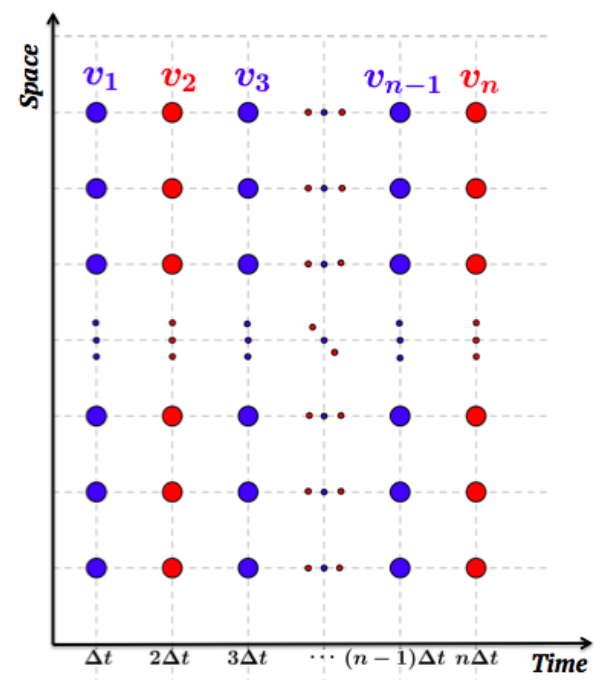

Figure 2. A schematic overview of the setup for the dynamic mode decomposition algorithm

For a nonlinear process, this assumption amounts to a linear tangent approximation. It allows us to formulate the data sequence as a Krylov sequence. Let $V_{i}^{j}$ denote the data sequence $\left[v_{i}, v_{i+1}, \ldots, v_{j}\right]$. On applying the matrix $A$ to each column of the data matrix $V_{1}^{n-1}$, we obtain the following relation by Eq. (12):

$$
A V_{1}^{n-1}=V_{2}^{n}=V_{1}^{n-1} S+r e_{n-1}^{T},
$$

where $S$ is a companion matrix, $\mathrm{r}$ is the residual vector, and $e_{n-1} \in \mathbb{R}^{n-1}$ is the $(n-1)$-th unit vector.

Denote the singular value decomposition of $V_{1}^{n-1}$ by $V_{1}^{n-1}=U \Sigma W^{*}$, where $\Sigma$ is a $r \times r$ diagonal matrix with $r=\operatorname{rank}\left(V_{1}^{n-1}\right)$. A low-order representation of $A$ can be obtained by mapping it to the proper orthogonal modes of $V_{1}^{n-1}$ as below:

$$
\tilde{A}:=U^{*} A U=U^{*} V_{2}^{n} W \Sigma^{-1}
$$

Alternatively, one can approximate the companion matrix $S$ with the standard least square procedures as below:

$$
S \approx R^{-1} Q^{H} V_{2}^{n}
$$

with $Q R=V_{1}^{n-1}$ as the economy-size QR-decomposition of the data sequence $V_{1}^{n-1} \cdot{ }^{15}$

The eigenvalues of both $\tilde{A}$ and $S$ can approximate some of the eigenvalues of $A$. Let $y_{i}$ be the $i$-th eigenvector of $\tilde{A}$ or $S$ with the corresponding eigenvalue being $\mu_{i}$. Then the eigenvalues $\mu_{i}$ describe the temporal evolution of the modes. In particular, the modal frequency $\omega_{i}$ and growth rate $\lambda_{i}$ can be calculated from the complex eigenvalues as below:

$$
\omega_{i}=\Im\left[\frac{\log \left(\mu_{i}\right)}{\Delta t}\right], \lambda_{i}=\Re\left[\frac{\log \left(\mu_{i}\right)}{\Delta t}\right]
$$

On obtaining the $i$-th eigenvectors $y_{i}$ of $\tilde{A}$ or $S$, the $i$-th dynamic mode can be constructed by $\phi_{i}=U y_{i}$ or $\phi_{i}=V_{1}^{n-1} y_{i}$, respectively. The flow field $v(\vec{x}, t)$ can then be approximated by a summation of the dynamic modes as below: 


$$
v(\vec{x}, t) \approx \bar{v}(\vec{x})+\sum_{i=1}^{n} c_{i} \phi_{i}(\vec{x}) e^{\left(\imath \omega_{i}+\lambda_{i}\right) t}
$$

where $\phi_{i}(\vec{x})$ represents the $i$-th DMD mode corresponding to the eigenvalue $\mu_{i}$, and $c_{i}$ is the amplitude of the $i$-th mode which can be determined from a reconstruction of the original data. In this study, we use the first snapshot to recover the amplitude. In this representation, the average flow field is singled out, as it represents the zero frequency mode.

It is assumed that the SVD-based DMD algorithm is more robust for noisy or contaminated data from experiments or simulations. ${ }^{15}$ By contrast, the least square based method is more straightforward to implement. In this paper, we use the least square based DMD algorithm, since the good quality of data as obtained from high order numerical simulation poses no difficulty for the analysis.

\section{Proper Orthogonal Decomposition}

Proper orthogonal decomposition is another important tool that can be used to extract coherent structures of the flow field. The representative development efforts within the context of fluid mechanics can be traced back to the classical POD ${ }^{29}$ and the snapshot POD. ${ }^{30}$ This type of method compresses the full data sets into a low-dimensional description comprised of a few dominant modes based on the orthogonal decomposition of the spatial velocity covariance matrix. By applying the method of snapshots ${ }^{30}$ the velocity field is decomposed into the time-mean and fluctuation components, i.e.,

$$
v(\vec{x}, t)=\bar{v}(\vec{x})+v^{\prime}(\vec{x}, t)
$$

where $\bar{v}$ and $v^{\prime}$ represent the time-averaged and fluctuation components of the velocity, respectively. Note that $v^{\prime}$ has zero mean value. We form the snapshots matrix of $v^{\prime}\left(\vec{x}, t_{i}\right), i=1, \ldots, N$, and compute the eigenvalues and eigenvectors of its temporal correlation matrix:

$$
C X=\lambda X
$$

where $C_{i j}=\int_{\Omega} v_{i}^{\prime} v_{j}^{\prime} d x$, and $v_{i}^{\prime}$ is the velocity fluctuation at $i$-th time instant. The $k$-th POD mode can be constructed by $\psi_{k}(\vec{x})=\sum_{i=1}^{N} X_{i k} v_{i}^{\prime}$.

Correspondingly, the flow field $v(\vec{x}, t)$ can be decomposed into a summation of spatial and temporal modes as below:

$$
v\left(\vec{x}, t_{i}\right) \approx \bar{v}(\vec{x})+\sum_{k=1}^{N} d_{k}\left(t_{i}\right) \psi_{k}(\vec{x})
$$

where $d_{k}\left(t_{i}\right)=\left(\psi_{k}, v_{i}^{\prime}\right)_{w}$, and $w$ is the weighting function that can be determined from the mesh.

The aforementioned two techniques share similarities, but they are apparently different from several aspects. The DMD modes are separated by frequency, whereas the POD modes are distinguished by energy ranking. Though both techniques can describe the evolution of coherent structures, they describes the temporal modes in different forms. Specifically, DMD characterizes the temporal modes with the term $e^{\left(\imath \omega_{i}+\lambda_{i}\right) t}$, whereas POD uses time-dependent mode amplitude coefficient with spatial modes mutually orthonormal with respect to an appropriate inner product. Previous studies have shown that there may exist dynamically highly relevant but zero-energy modes in unsteady flow system. ${ }^{28}$ As a result, modification to POD is needed to capture transient features in a dynamical system. In the DMD method, the modes and frequencies are determined 
without relying on an inner product, which makes it easily applicable to flow quantities at any spatial locations. ${ }^{31}$ In this study, we compare the performance of DMD and POD on the analysis of wake structures behind oscillating square cylinders.

\section{Results}

Four types of wake structures behind oscillating square cylinders are considered in this section. These cases include:

- Long-range oscillatory vortex street $(R e=100$ and $S t=0.12)$;

- Regular von Kármán vortex street $(R e=100$ and $S t=0.18)$;

- Asymmetric vortex street $(R e=400$ and $S t=0.12)$; and

- Dynamic multi-vortex wake $(R e=400$ and $S t=0.054)$.

Snapshots of vorticity fields for the four cases from CFD simulation are presented in Figure 3. DMD analysis is applied to all these vorticity fields. POD analysis is applied to the long-range oscillatory vortex street and the dynamic multi-vortex wake for comparison.

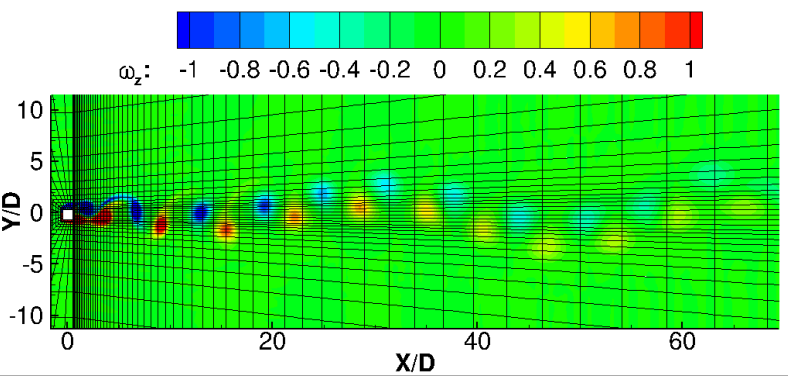

(a)

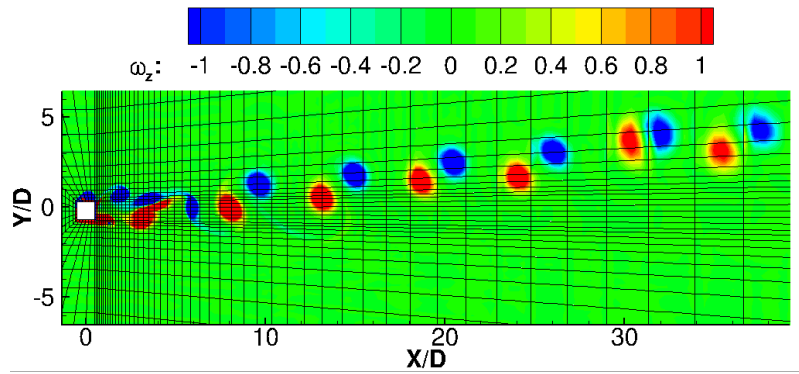

(c)

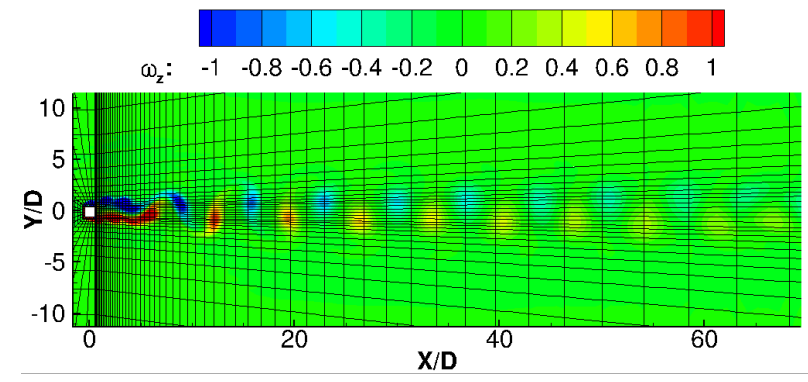

(b)

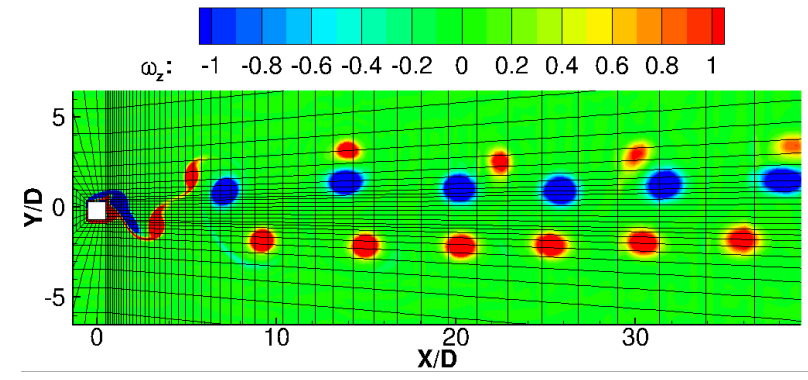

(d)

Figure 3. Vorticity fields from CFD simulation for (a) $R e=100$ and $S t=0.12 ;$ (b) $R e=100$ and $S t=0.18 ;(\mathbf{c})$ $R e=400$ and $S t=0.12 ;$ (d) $R e=400$ and $S t=0.054$.

\section{VI.A. Long-range oscillatory vortex street}

Results with $R e=100$, and $S t=0.12$ are discussed in this section. A total number of 224 snapshots are used in the analysis. This corresponds to two oscillating cycles. The time interval $\Delta t$ between two successive snapshots is 0.015. Results for the DMD spectral analysis is presented in Figure 4. In part (a), the eigenvalues $\mu_{i}$, which are also called Ritz values, ${ }^{14}$ are found to be distributed very close to the unit circle in the complex plane. This indicates the growth/decay rate of the flow structures is close to zero. In part (b), the growth rates $\lambda_{i}$ of the DMD modes are plotted against 
the frequency $\omega_{i}$. It is observed that the absolute values of the growth rate are very small, and positive and negative values implies that the corresponding DMD modes are in the growing and decaying states, respectively. Note that only the time-averaged state (i.e., 0-th mode) and the first four pairs of DMD modes are shown here. Since we have normalized the DMD modes, $\left|c_{i}\right|$ gives the magnitude of the $n$-th mode. In part (c), the amplitude $\left|c_{i}\right|$ of DMD modes are drawn against the frequency $\omega_{i}$ to illustrate the relative strength of each mode. There is one distinct peak at zero frequency, which indicates the time-averaged state, in the energy spectrum over a range of frequencies. The same set of dominant DMD modes are marked in all subfigures to establish the correspondence of different properties of the same mode.

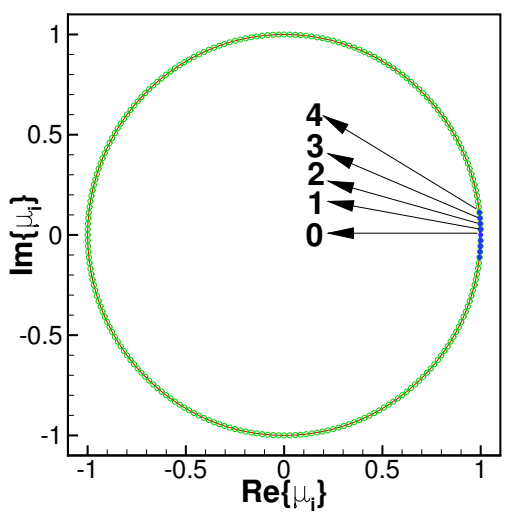

(a)

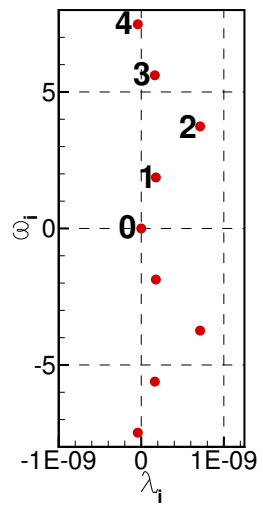

(b)

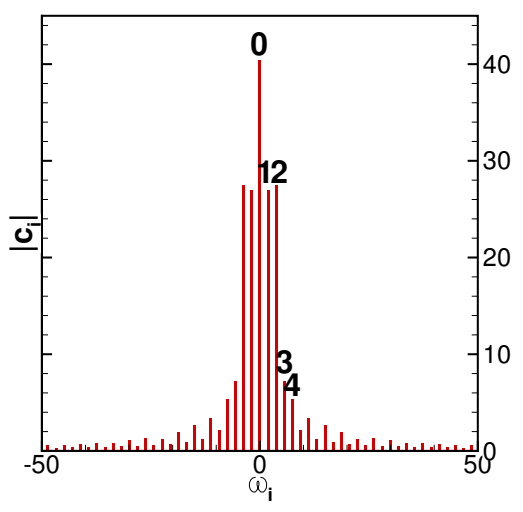

(c)

Figure 4. Results of DMD analysis of a $\mathrm{N}=\mathbf{2 2 4}$ snapshot sequence of wake structure behind an oscillating square cylinder at $R e=100$ and $S t=0.12$ : (a) real and imaginary parts of the eigenvalues; (b) DMD spectrum; (c) DMD amplitude spectrum.

Next, we present the dominant DMD modes, as marked in Figure 4 to aid the wake structure analysis. The time-averaged vorticity field is shown in Figure 5, which represents the 0-th DMD and POD mode. The DMD mode are ranked by frequency, i.e., $\left|\omega_{i}\right|$. The spatial distribution of
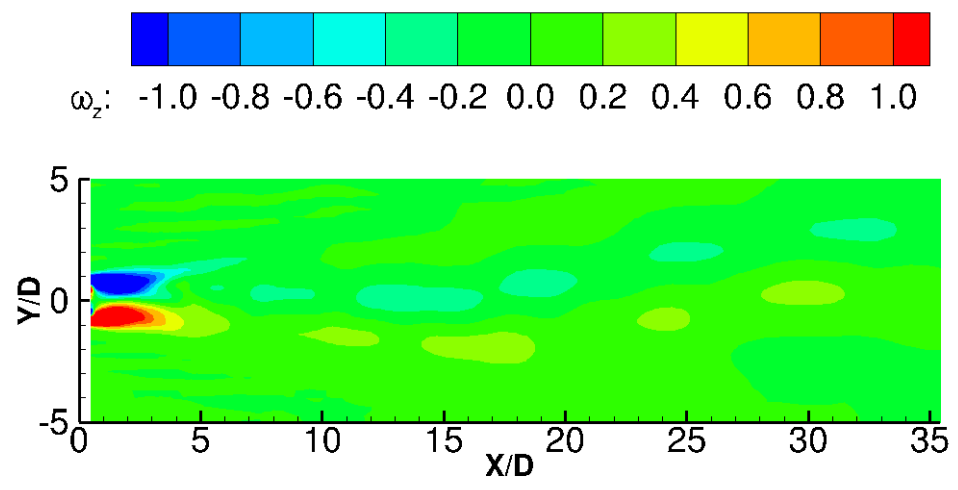

Figure 5. Time-averaged vorticity field at $R e=100$ and $S t=0.12$

the real parts of the numbered DMD modes are shown in Figure 6. The imaginary parts can be presented in the same way. They are not shown here for conciseness.

As can be seen from Eq. 17, the amplitude $c_{i}$ of the $i$-th DMD mode can be reconstructed from the original data. In this study, $c_{i}$ is calculated from the first snapshot, i.e., $v_{1}$. As a result, the flow field at any time $t$ can be reconstructed using Eq. 17. A typical reconstructed instantaneous vorticity field using the zero and first four pairs of DMD modes is plotted in Figure 7. The contours 

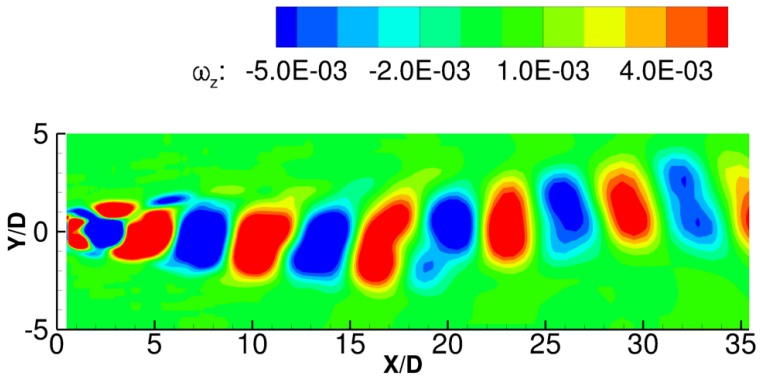

(a)
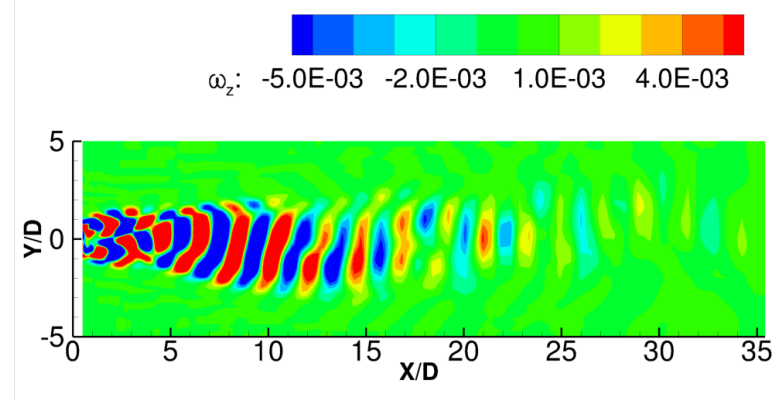

(c)
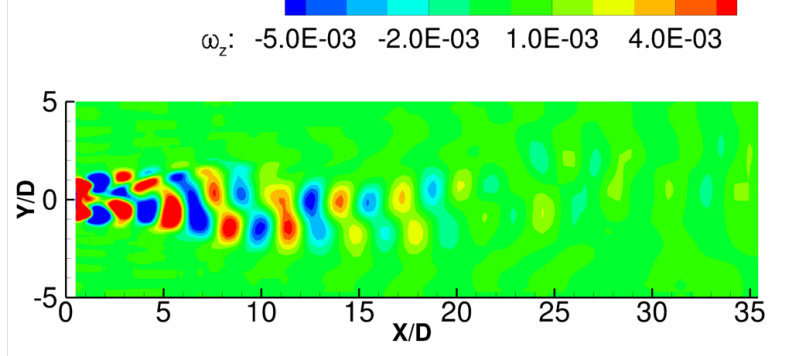

(b)

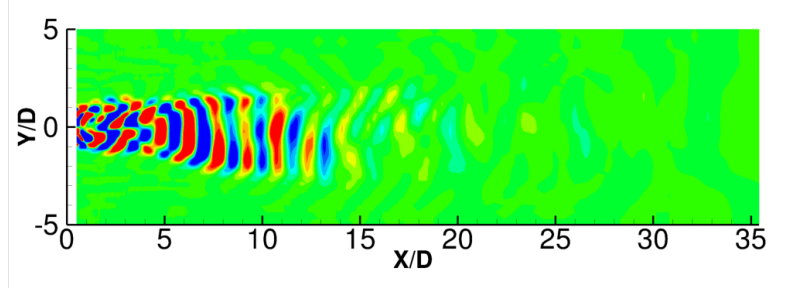

(d)

Figure 6. Real part of the temporal DMD modes for $R e=100$ and $S t=0.12$ : (a) mode 1 ; (b) mode 2 ; (c) mode $3 ;$ (d) mode 4.

of the vorticity field at the same time instant directly from the numerical simulation is also plotted in this figure for comparison. A good visual agreement is obtained from these two sets of data. This demonstrates that four pairs of low frequency modes plus the zero mode are adequate to give a good representation of the vortex dynamics in this case.

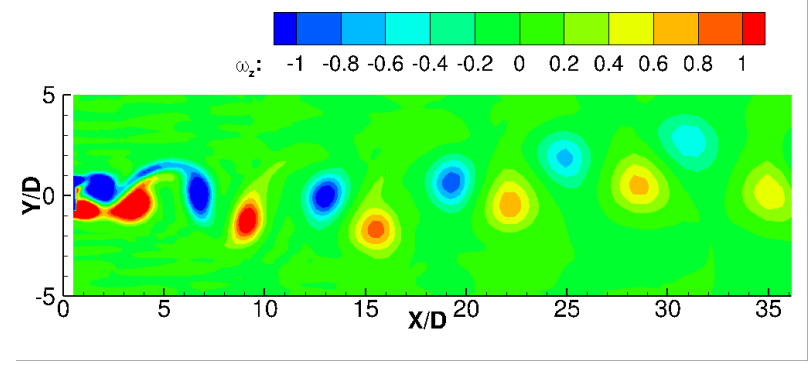

(a)

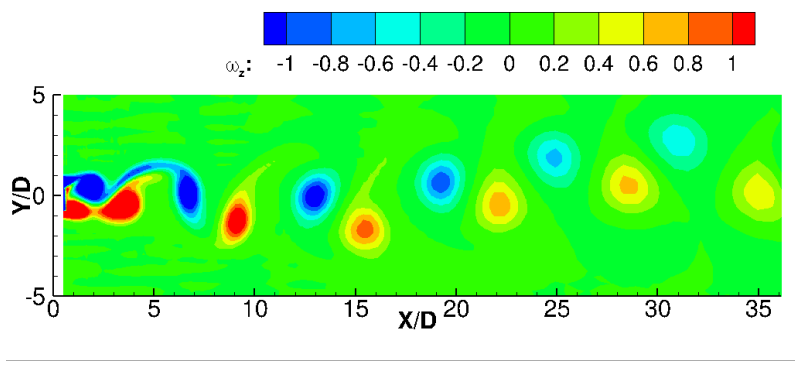

(b)

Figure 7. A typical instantaneous vorticity field $\left(t=t_{0}+75 \times \Delta t\right)$ for $R e=100$ and $S t=0.12$ : (a) reconstruction using 4 pairs of dominant DMD modes plus the zero mode; (b) original vorticity field from the numerical simulation at the same time instant.

POD analysis is made based on the same sequence of snapshots as those used in DMD analysis. For comparison, we also show the reconstructed flow field at the same time instant using the leading eight POD modes in Figure 8. It is clear that the leading eight dominant pod modes also represents the original vorticity field very well. More insights can be gained from the eigenvalues corresponding to these modes. As seen from Figure 9, the eigenvalues of the first four modes are 


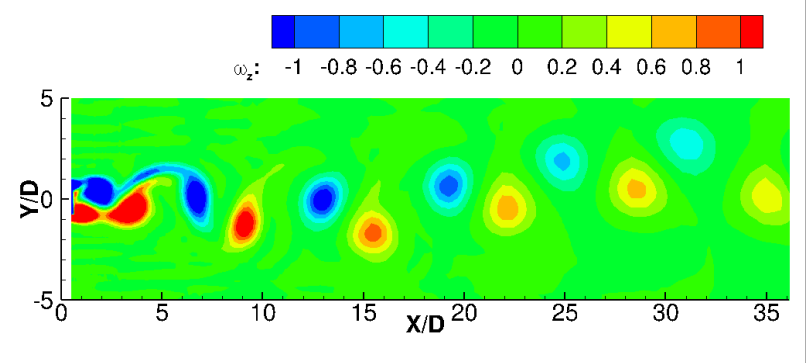

(a)

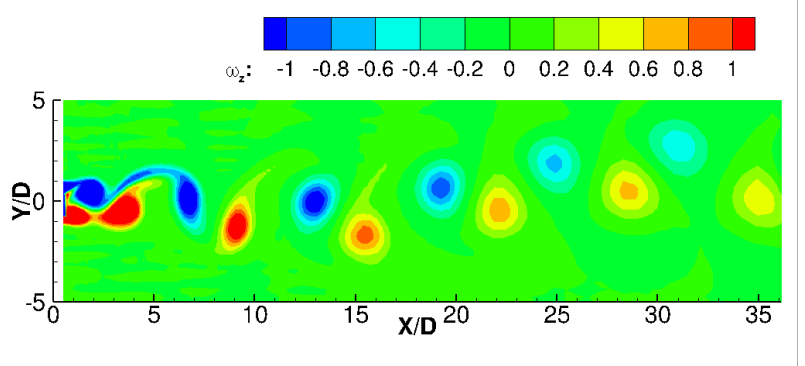

(b)

Figure 8. A typical instantaneous vorticity field $\left(t=t_{0}+75 \times \Delta t\right)$ for case $R e=100$ and $S t=0.12$ : (a) reconstruction using the leading eight dominant POD modes plus the zero mode; (b) original vorticity field from the numerical simulation at the same time instant.

much larger than those of the remaining modes, and fast decay is observed as the mode indices increase. The cumulative distribution is calculated by the summation of the normalized eigenvalues of the corresponding modes. The curve indicates that the leading eight modes contributes to above $95 \%$ of the total value. We show the corresponding POD modes in Figure 10. Compared with Figure 6, it is found that the dominant POD modes are largely consistent with the dominant DMD modes. This indicates that for the long-range oscillatory wake, the dominant low frequency modes identified by the DMD technique are also dominant in energy contribution as measured in the sense of POD analysis.

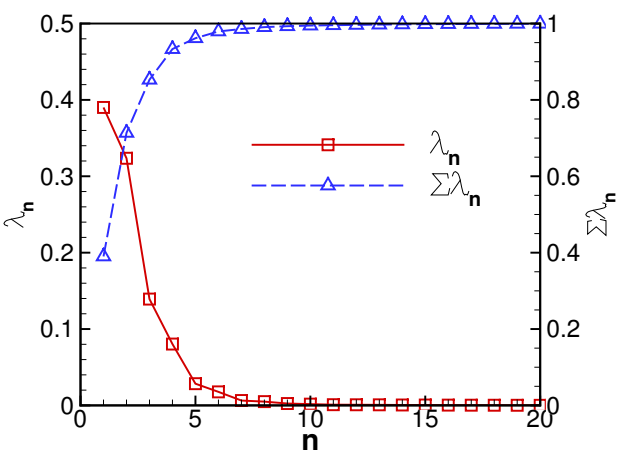

Figure 9. Normalized eigenvalues and cumulative distribution of pod modes for case $R e=100$ and $S t=0.12$.

\section{VI.B. Regular von Kármán vortex street}

In this section, we show results from DMD analysis for the case with $R e=100$ and $S t=0.18$. The DMD spectrum is presented in Figure 11. Compared with the long-range oscillatory vortex street case $(R e=100$ and $S t=0.12)$, the lowest angular frequency of the dominate modes increases from $\sim 1.87$ to $\sim 2.55$. If $S t$ number is used to nondimensionalize the frequency, the lowest $S t$ number for the long-range oscillatory vortex street case is $\sim 0.12$ and that for the regular von Kármán vortex street case is $\sim 0.16$. Apparently, the long-range oscillatory vortex street case falls into the 'lock-in' regime, but the regular von Kármán vortex street case does not.

The time-averaged vorticity field (i.e., zero mode) is shown in Figure 12. The spatial patterns of the first four pairs (ranked by frequency) of DMD modes are illustrated in Figure 13. Using the leading 4 pairs of dominant DMD modes and the zero mode, the vorticity field can be faithfully reconstructed. As demonstrated in Figure 14, there is no apparent difference in the vorticity field 

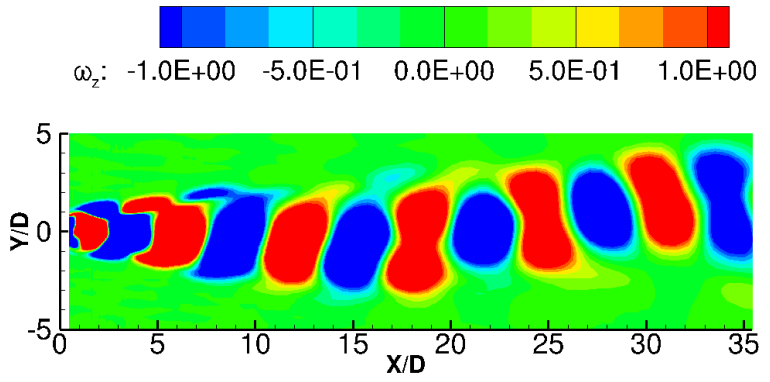

(a)
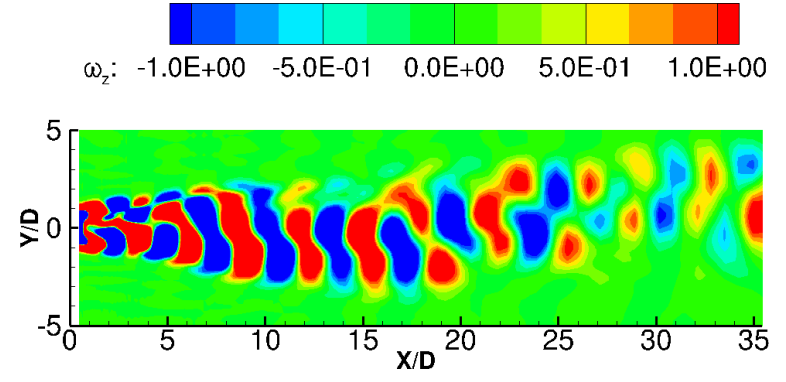

(c)

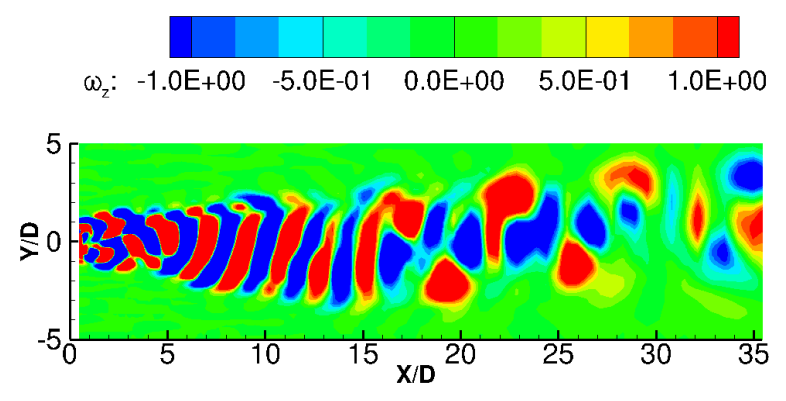

(e)
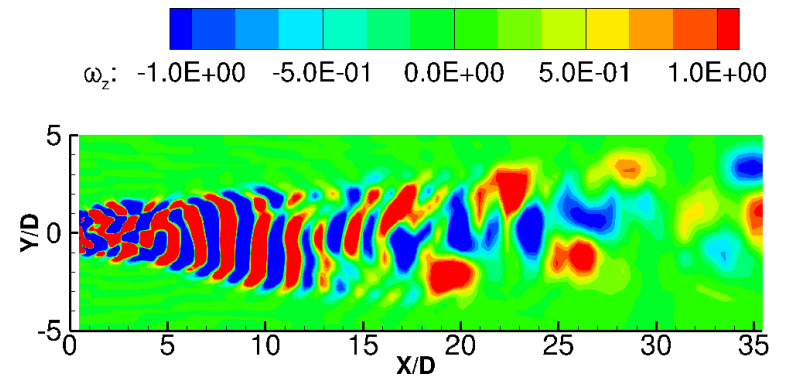

(g)
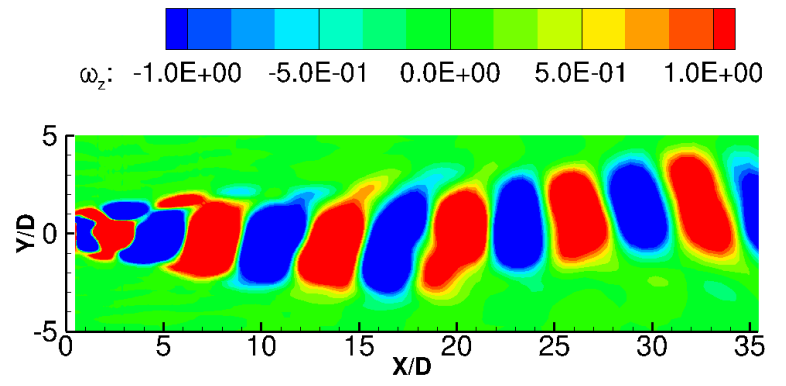

(b)

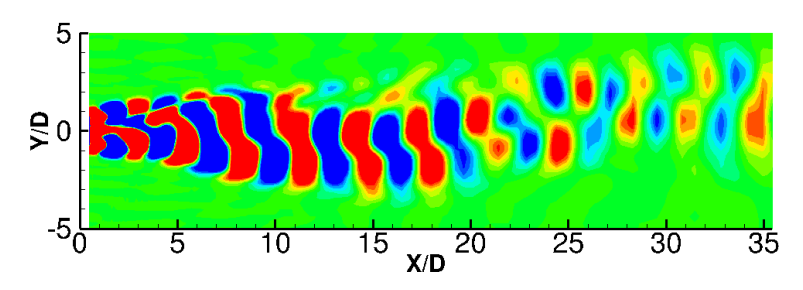

(d)
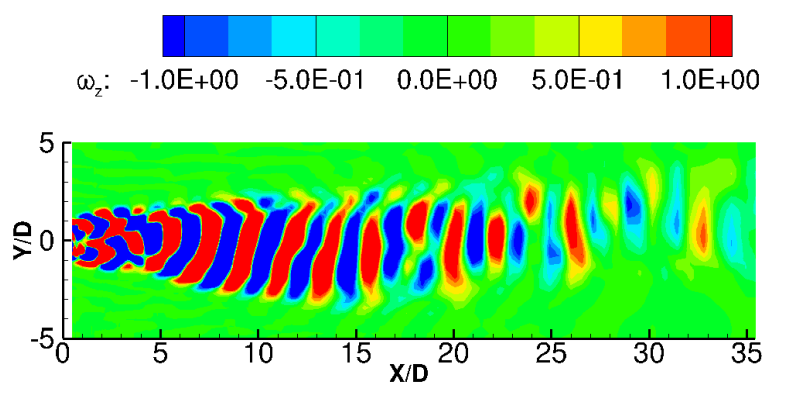

(f)
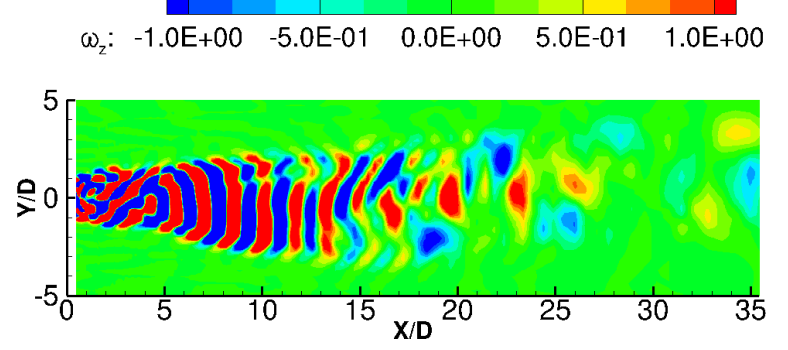

(h)

Figure 10. Spatial patterns of the POD modes for case $R e=100$ and $S t=0.12$ : (a) $\operatorname{mode} 1$; (b) mode 2 ; (c) mode 3 ; (d) mode 4 ; (e) mode 5 ; (f) mode 6 ; (g) mode 7 ; (h) mode 8. 
between the reconstructed and original data for a typical time instant during the oscillation cycle. Results from POD analysis show similar trend when compared with those from DMD analysis. We omit the POD results for this case for conciseness.

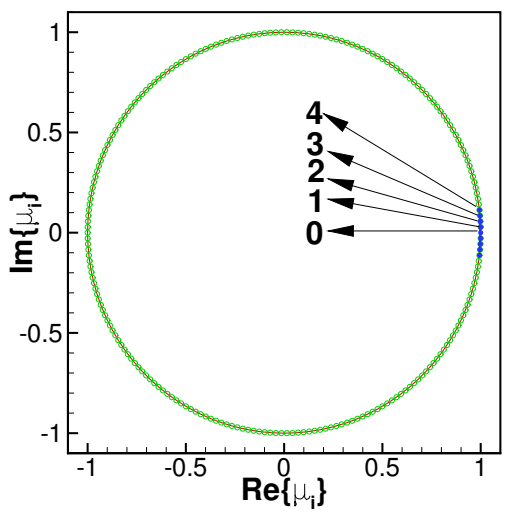

(a)

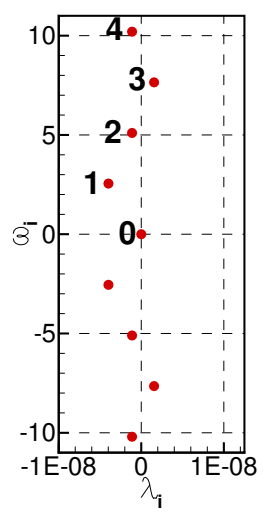

(b)

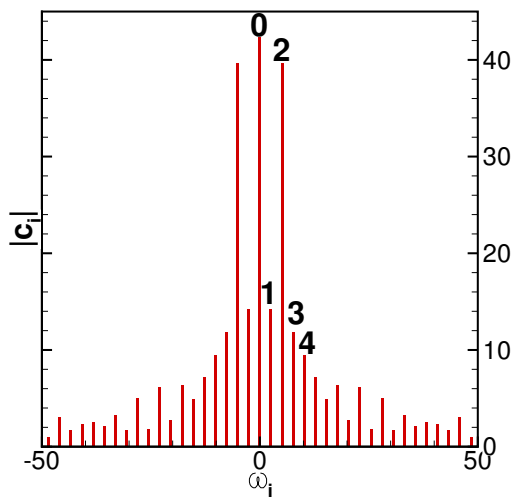

(c)

Figure 11. Results of DMD analysis of a $\mathrm{N}=222$ snapshot sequence of wake structure behind an oscillating square cylinder at $R e=100$ and $S t=0.18$ : (a) real and imaginary parts of the eigenvalues; (b) DMD spectrum; (c) DMD amplitude spectrum.
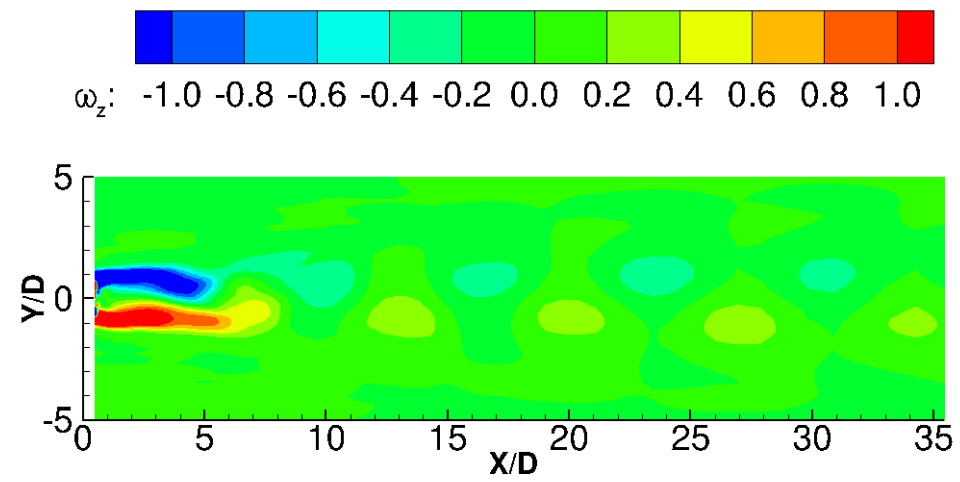

Figure 12. Time-averaged vorticity field at $R e=100$ and $S t=0.18$

\section{VI.C. Asymmetric vortex street}

Now we study the effect of Reynolds number on the topology of wake structures behind the oscillating square cylinder. The Strouhal number $S t$ is selected as 0.12, and the Reynolds number Re is increased to 400. In Figure 15, we show the DMD spectrum. Similarly as in previous cases, the Ritz values are relatively uniformly distributed around the unit circle in the complex plane in this case. The low growth/decay rate indicates that the dominant low frequency modes are very close to neutral states. The lowest frequency of the dominant DMD modes remains to be $\sim 1.87$ as in the case $R e=100$ and $S t=0.12$. This indicates that the frequency of the dominant modes are mainly determined by the Strouhal number.

The time-averaged vorticity field is shown in Figure 16. The spatial patterns of the first four pairs of DMD modes are presented in Figure 17. As observed from the Figure 17, the spatial patterns of the DMD modes share similarities with those at $R e=100$ and $S t=0.12$ for each distinct frequency. But the wake turns to be asymmetric about the horizontal line, which appears 


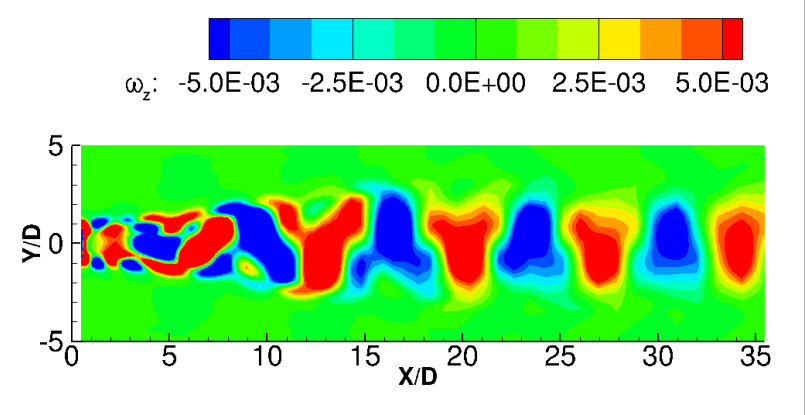

(a)

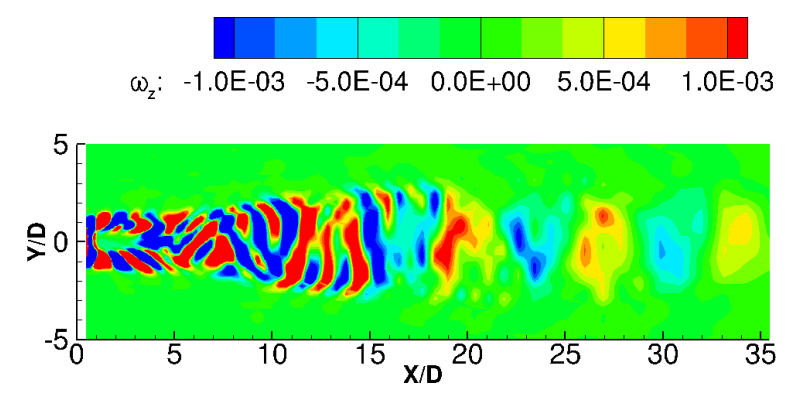

(c)
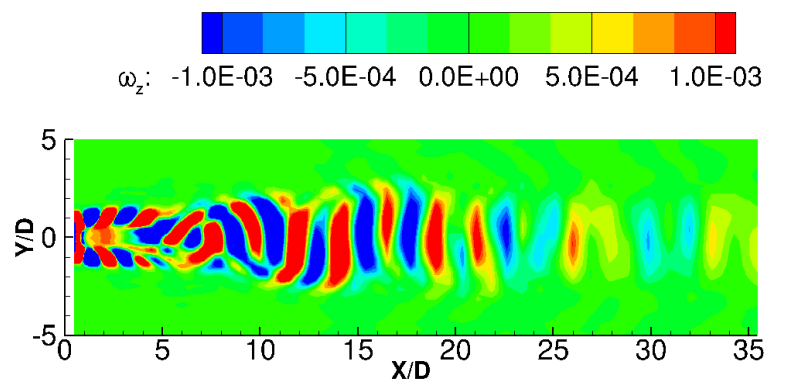

(b)

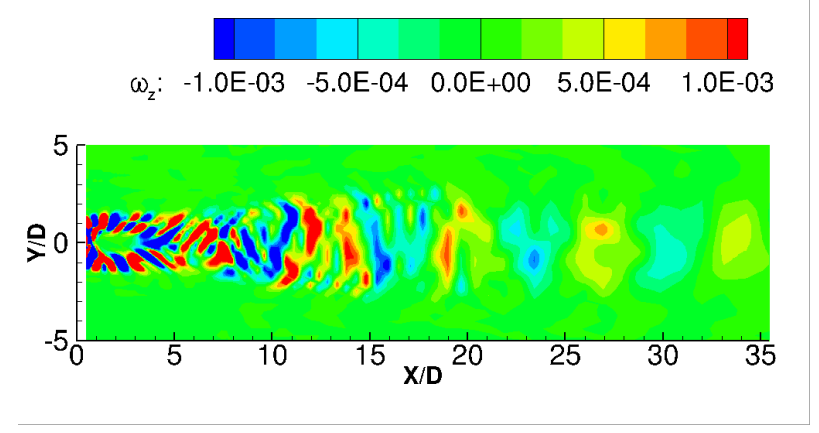

(d)

Figure 13. Real part of the temporal DMD modes for $R e=100$ and $S t=0.18$ : (a) $\operatorname{mode} 1$; (b) $\operatorname{mode} 2$; (c) mode $3 ;$ (d) mode 4 .

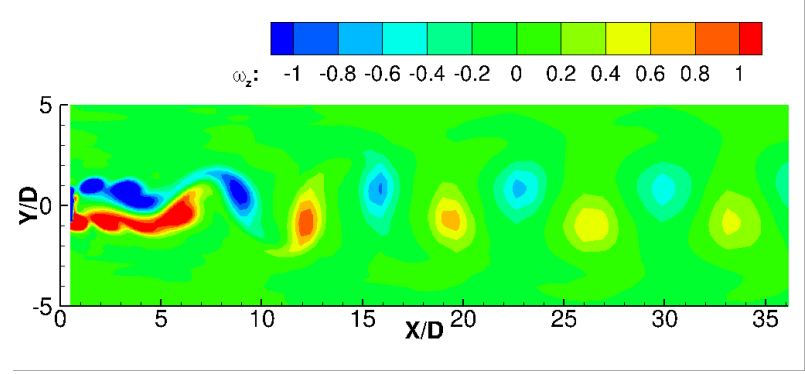

(a)

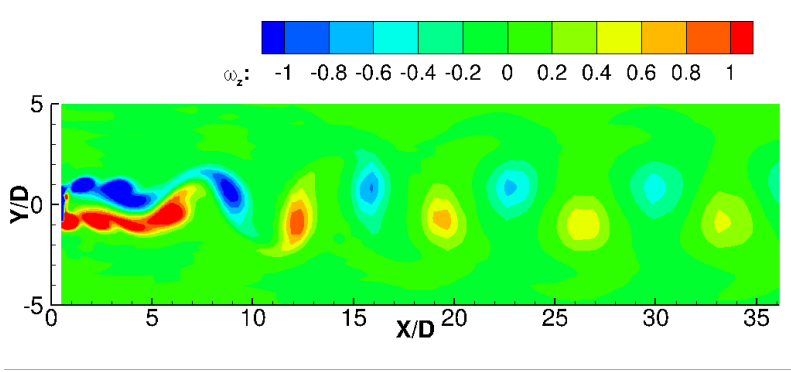

(b)

Figure 14. A typical instantaneous vorticity field $\left(t=t_{0}+75 \times \Delta t\right)$ for $R e=100$ and $S t=0.18$ : (a) reconstruction using 4 pairs of dominant DMD modes plus the zero mode; (b) original vorticity field from the numerical simulation at the same time instant.

to be the effect of higher Reynolds number. Note that this vortex street pattern is different from the long-range oscillatory vortex street pattern at $R e=100$ and $S t=0.12$. In that case, the wake shows large-scale wavy structure. But in the current case, the direction of the reflected wake does not change. This agrees with previous findings of $\mathrm{Yu}$ et al. on asymmetric wake vortex structures around an oscillating airfoil. ${ }^{27}$

Another observation is that the energy of the entire flow field is spread over a wider frequency range (as seen in Figure 15 part (c)), as the Reynolds number increases. In order to give a good representation of the original vorticity field, we need to include more dominate mode pairs in the reconstruction. Figure 18 shows results from sixteen pairs of dominant modes plus the zero mode. It is observed from this figure that good agreement with the original field is obtained when we 
include necessary amount of dominant modes.

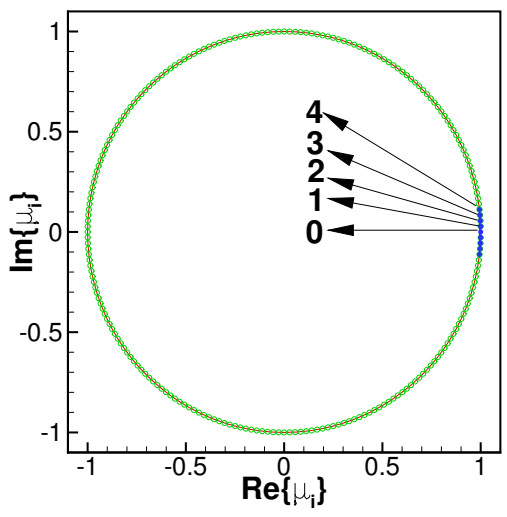

(a)

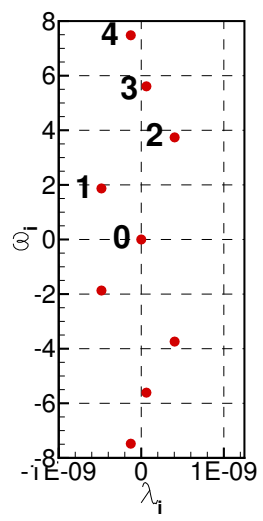

(b)

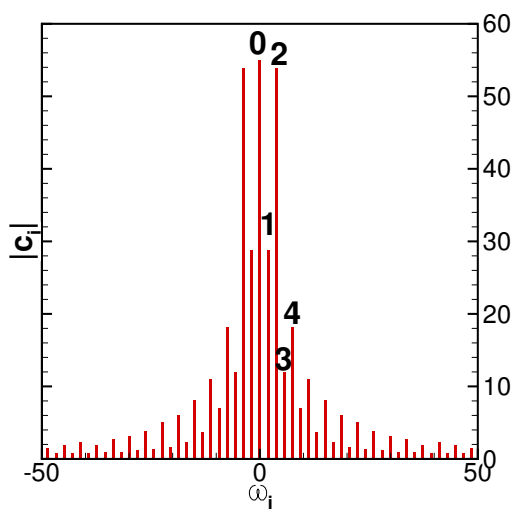

(c)

Figure 15. Results of DMD analysis of a $\mathrm{N}=224$ snapshot sequence of wake structure behind an oscillating square cylinder at $R e=400$ and $S t=0.12$ : (a) real and imaginary parts of the eigenvalues; (b) DMD spectrum; (c) DMD amplitude spectrum.
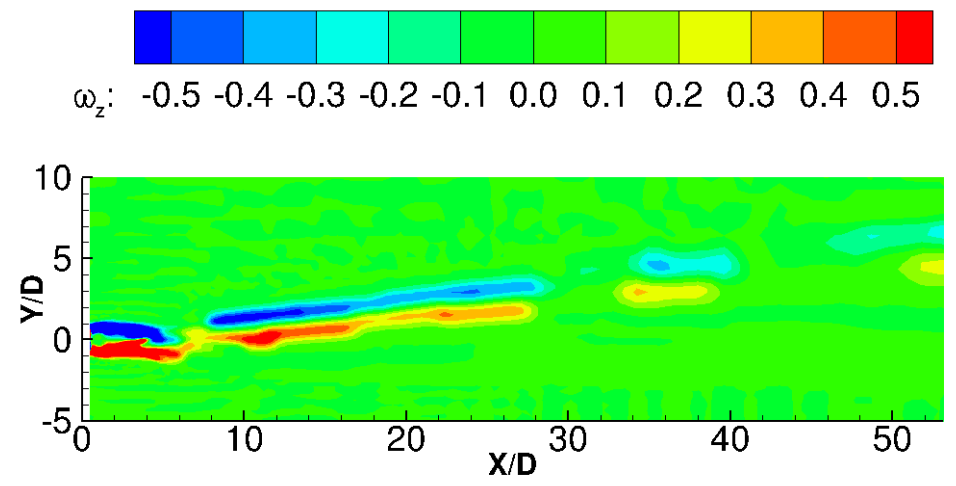

Figure 16. Time-averaged vorticity field at $R e=400$ and $S t=0.12$

\section{VI.D. Dynamic multi-vortex wake}

Finally, we show wake analysis results at $R e=400$ and $S t=0.054$. This case represents interesting wake dynamics, which features a third array of vortices first entrained into and then ejected from the main vortex street as traveling along the downstream direction. Figure 19 gives the spectral analysis of the DMD modes. The Ritz values distribution (Figure 19, part (a)) and growth rate of the low frequency modes (Figure 19, part (b)) are largely the same as in previous cases. However, in the frequency-amplitude chart, we notice a tiny contribution from the odd low frequency modes as marked in Figure 19, part (c). In particular, we have displayed the time-averaged vorticity field and the DMD modes in Figure 20 and Figure 21, respectively. As interestingly as the odd modes present themselves, these dynamics contribute very little to the whole vorticity field during the reconstruction process. So we need to use more pairs of low frequency DMD modes plus the zero mode (Figure 16) to obtain a good representation of the original field. The results for sixteen pairs is shown in Figure 22.

We also conduct POD analysis for the same data sequence in this case. As seen from Figure 25, though the even modes share similar spatial patterns to those from DMD analysis, the dynamics 

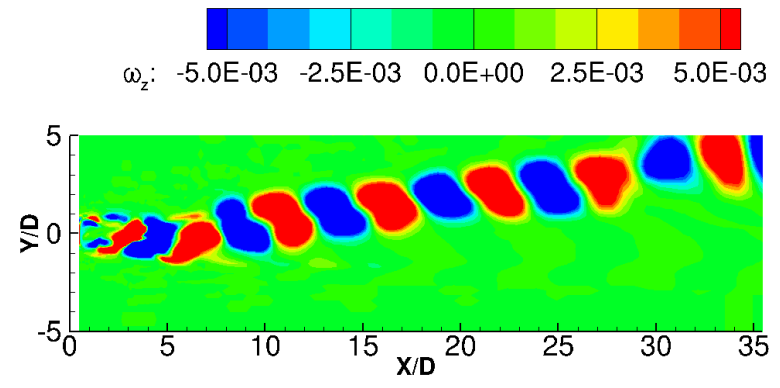

(a)

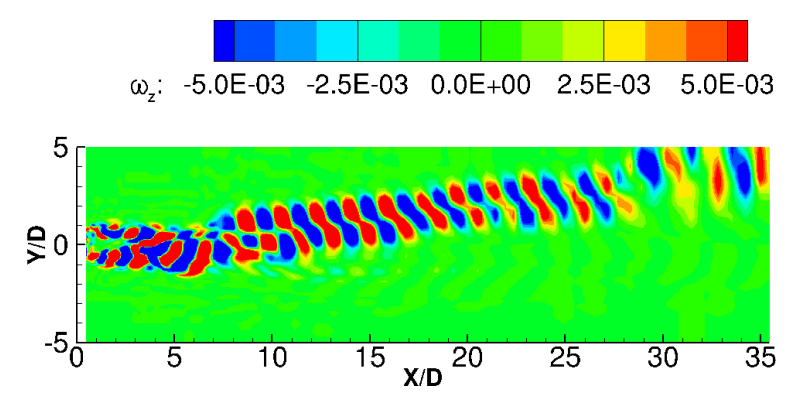

(c)
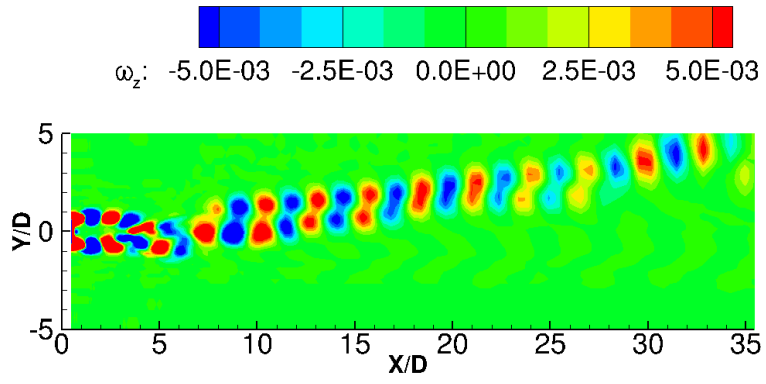

(b)
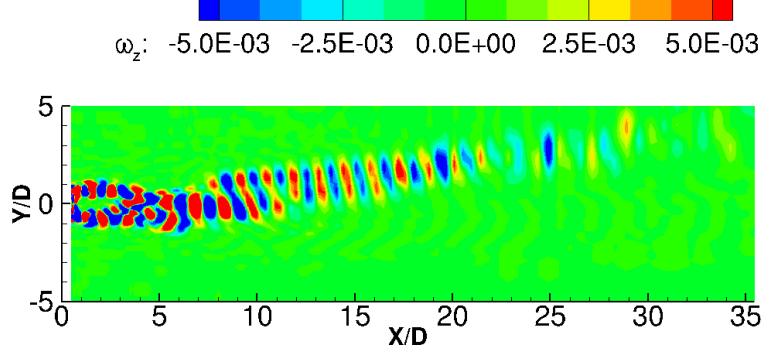

(d)

Figure 17. Real part of the temporal DMD modes for $R e=400$ and $S t=0.12$ : (a) $\operatorname{mode} 1$; (b) $\operatorname{mode} 2$; (c) mode $3 ;$ (d) mode 4 .

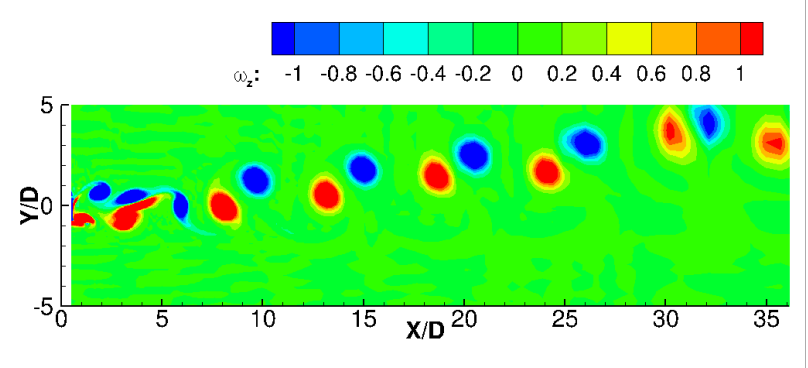

(a)

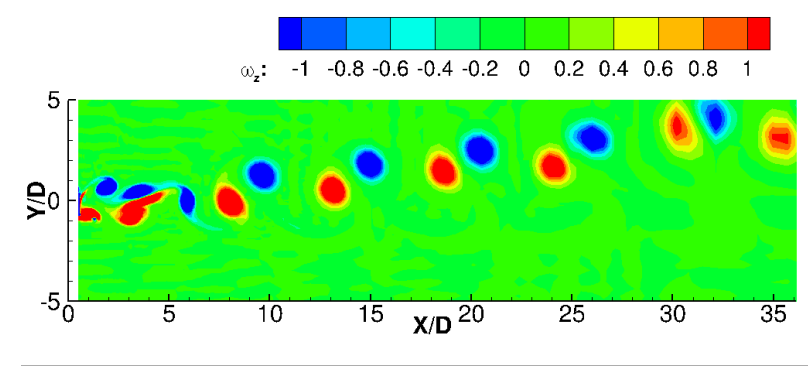

(b)

Figure 18. A typical instantaneous vorticity field $\left(t=t_{0}+75 \times \Delta t\right)$ for $R e=400$ and $S t=0.12$ : (a) reconstruction using 16 pairs of dominant DMD modes plus the zero mode; (b) original vorticity field from the numerical simulation at the same time instant.

of the odd leading DMD modes are not captured in the leading pod modes due to their low energy content. It can be observed from Figure 24 that the first eight POD modes contributes to above $95 \%$ of the total value. Thus, it is no surprise that the reconstructed vorticity field (Figure 23) from eight POD modes is non-distinguishable from that (Figure 22) from much more DMD modes.

From the comparison between DMD and POD analysis, it is found that the odd leading DMD modes have similar features to the dynamically highly relevant but zero-energy modes mentioned by Schmid. ${ }^{15}$ These dynamic modes may contribute to the development of robust low-dimensional models as presented by Noack et al. ${ }^{28}$ 


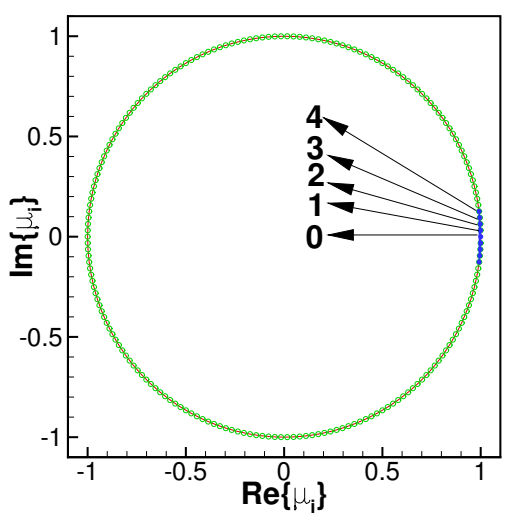

(a)

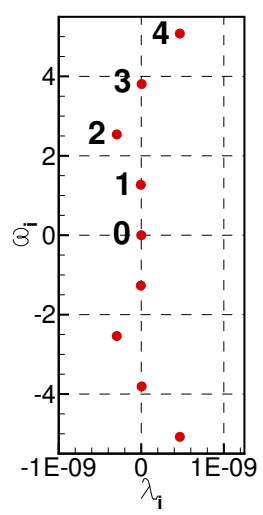

(b)

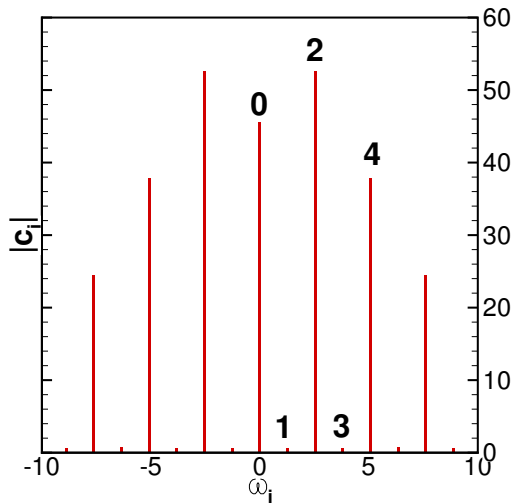

(c)

Figure 19. Results of DMD analysis of a $\mathrm{N}=198$ snapshot sequence of wake structure behind an oscillating square cylinder at $R e=400$ and $S t=0.054$ : (a) real and imaginary parts of the eigenvalues; (b) DMD spectrum; (c) DMD amplitude spectrum.
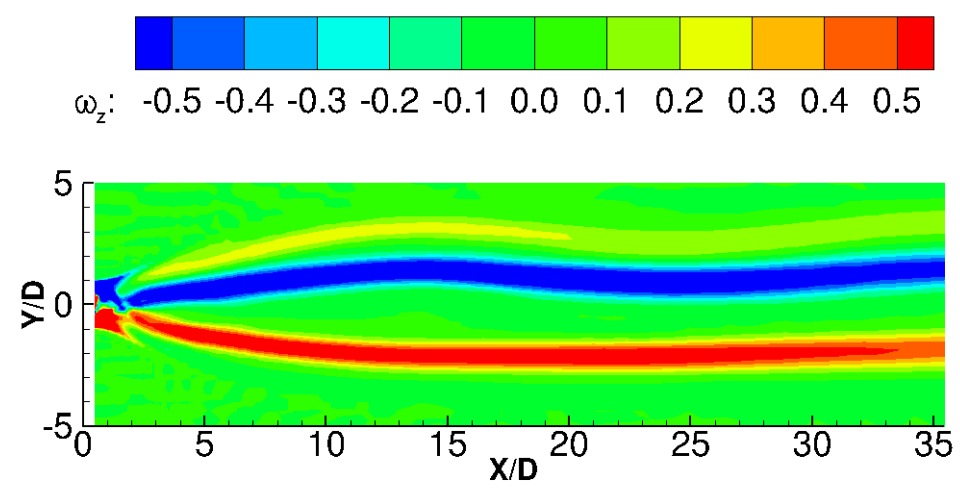

Figure 20. Time-averaged vorticity field at $R e=400$ and $S t=0.054$ 


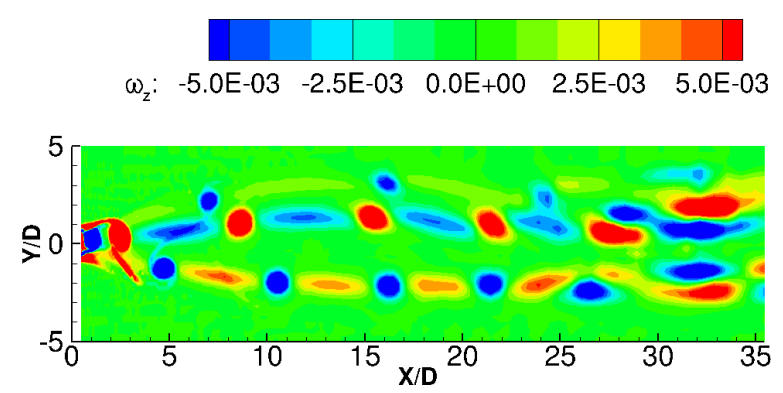

(a)

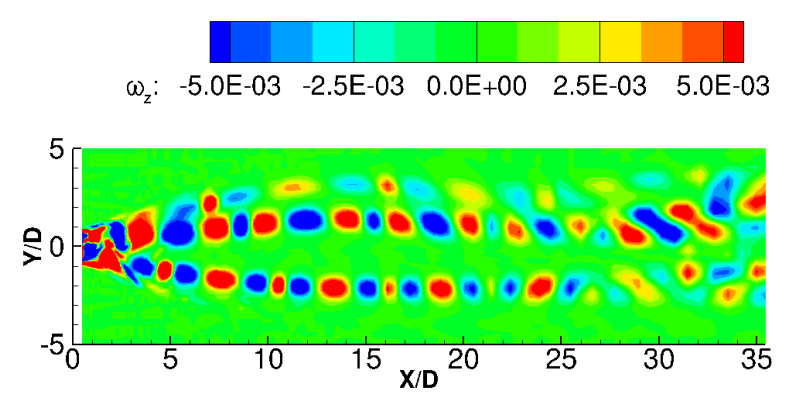

(c)
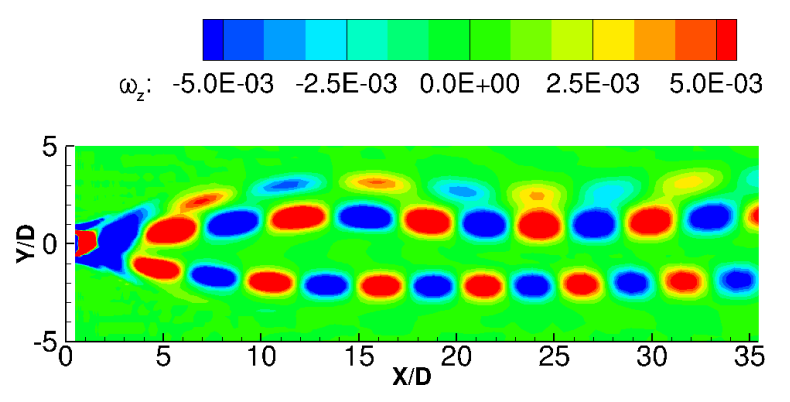

(b)
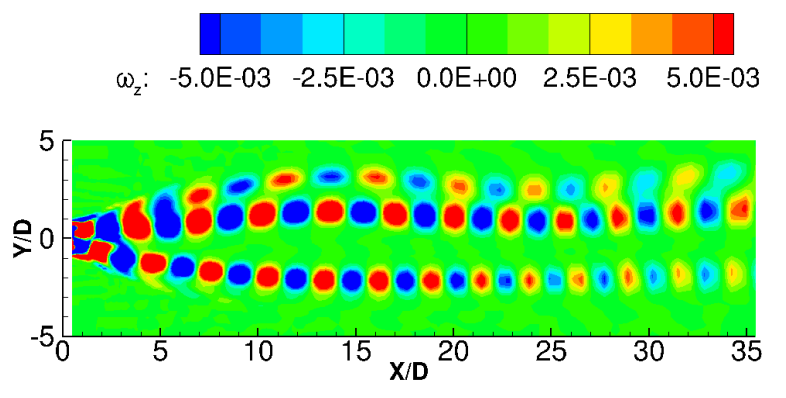

(d)

Figure 21. Real part of the temporal DMD modes for $R e=400$ and $S t=0.054$ : (a) mode 1 ; (b) mode 2 ; (c) mode $3 ;$ (d) mode 4 .

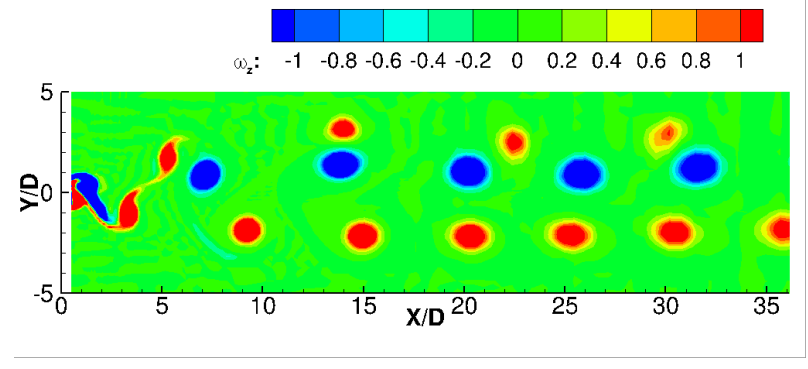

(a)

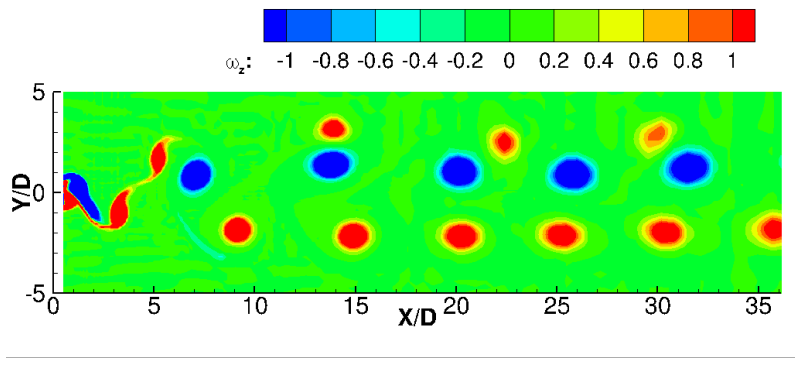

(b)

Figure 22. A typical instantaneous vorticity field $\left(t=t_{0}+75 \times \Delta t\right)$ for $R e=400$ and $S t=0.054$ : (a) reconstruction using 16 pairs of dominant DMD modes plus the zero mode; (b) original vorticity field from the numerical simulation at the same time instant. 


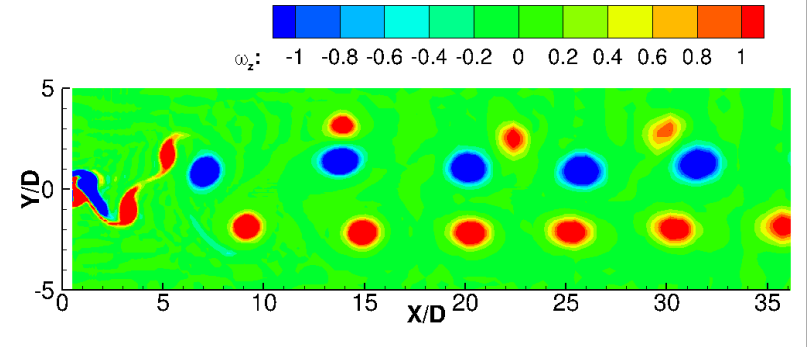

(a)

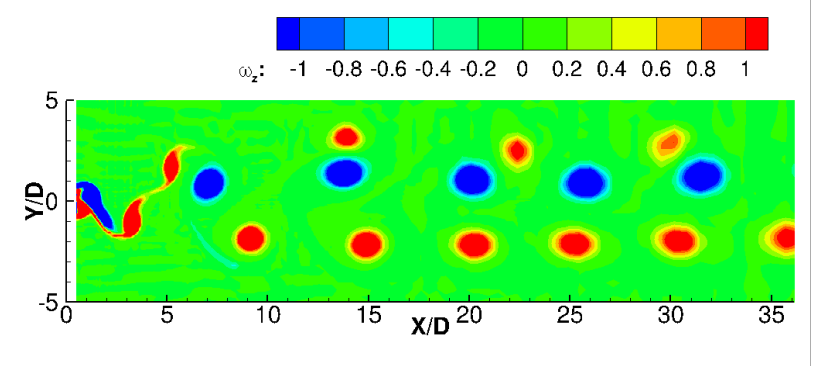

(b)

Figure 23. A typical instantaneous vorticity field $\left(t=t_{0}+75 \times \Delta t\right)$ for case $R e=400$ and $S t=0.054:$ (a) reconstruction using the leading 16 dominant POD modes plus the zero mode; (b) original vorticity field from the numerical simulation at the same time instant.

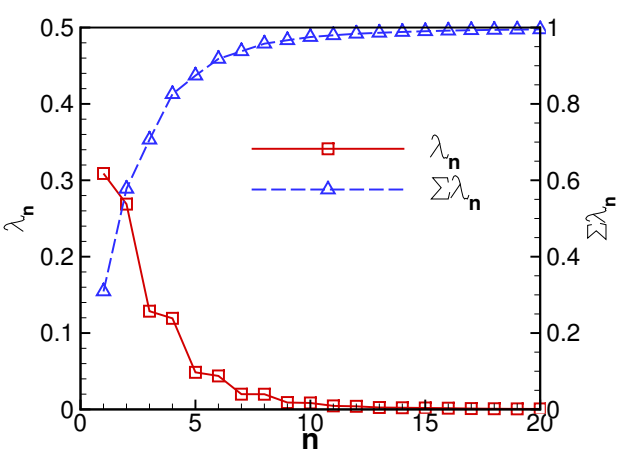

Figure 24. Normalized eigenvalues and cumulative distribution of pod modes for case $R e=400$ and $S t=0.054$.

\section{Conclusion}

In this study, the DMD technique is used to analyze wake structures behind oscillating square cylinders based on numerical simulation results. We conduct series of high-order CFD simulation at different Strouhal numbers and Reynolds numbers, extract data in the wake region, and analyze the dominant dynamic modes with the DMD technique in terms of vortex topology, frequency, growth rate, and amplitude. The DMD modes are compared with POD modes. The effects of Strouhal and Reynolds number on the dynamics of wake structures behind oscillating square cylinders are then revealed based on the mode decomposition analysis.

For most vortex street types studied, including the long-range oscillatory wake, regular von Kármán wake, and the asymmetric wake, DMD modes share much similarity with the POD modes. For a very dynamic wake, i.e., the so-called dynamic multi-vortex wake, the DMD technique captures certain dynamically highly relevant but low-energy modes. These modes are closely related to the transient effect presented in the evolution of the vortex wake. Further research is needed to study their implications on reduced-order modeling. 


\section{$\omega_{z}:-1.0 \mathrm{E}+00 \quad-5.0 \mathrm{E}-01 \quad 0.0 \mathrm{E}+00 \quad 5.0 \mathrm{E}-01 \quad 1.0 \mathrm{E}+00$}

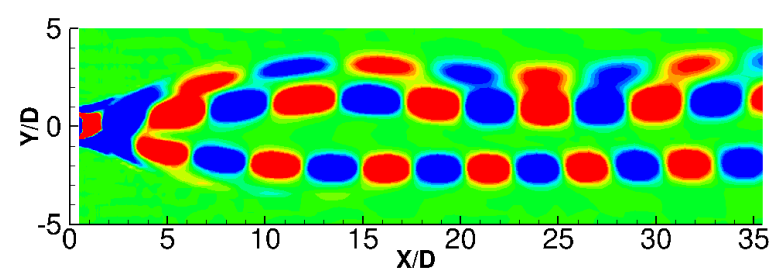

(a)
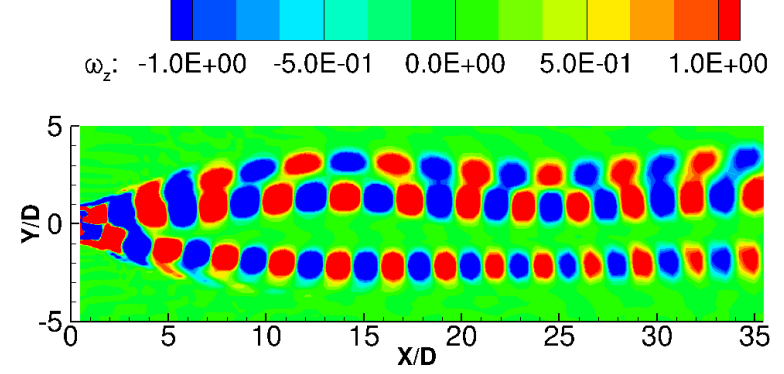

(c)

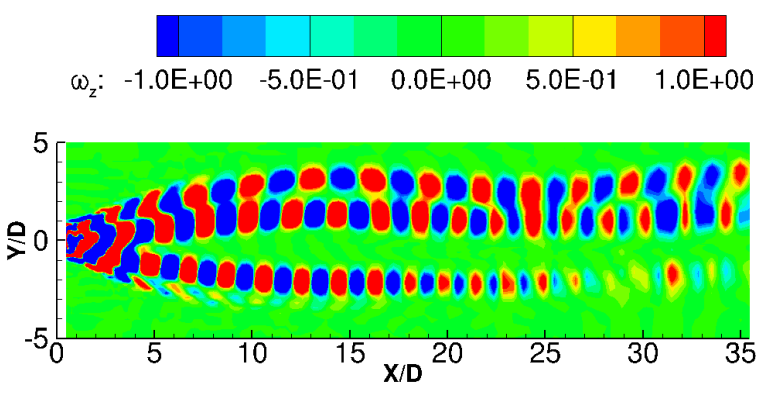

(e)
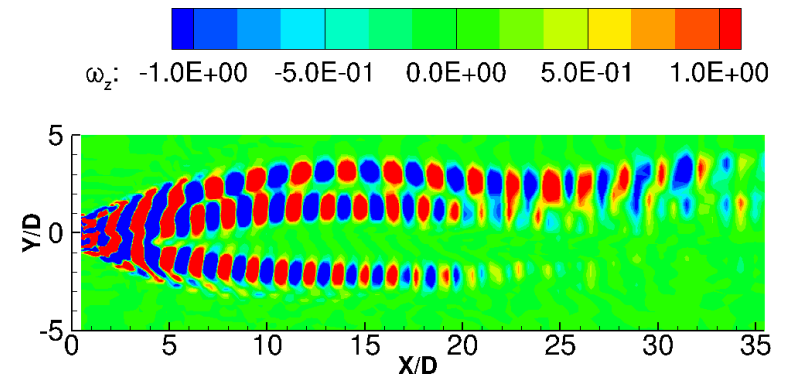

(g)
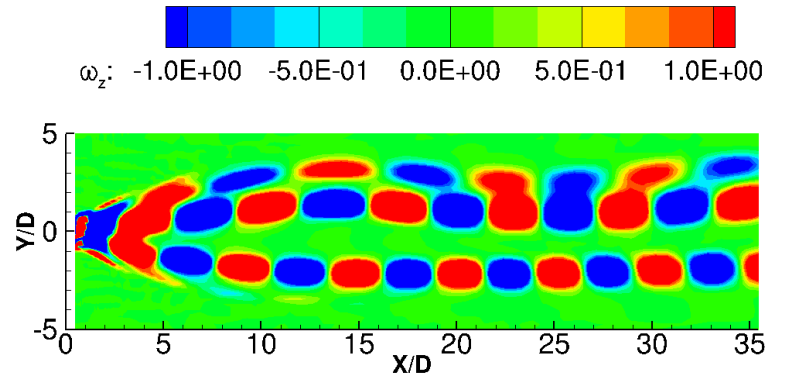

(b)

$$
\omega_{z}:-1.0 \mathrm{E}+00 \quad-5.0 \mathrm{E}-01 \quad 0.0 \mathrm{E}+00 \quad 5.0 \mathrm{E}-01 \quad 1.0 \mathrm{E}+00
$$

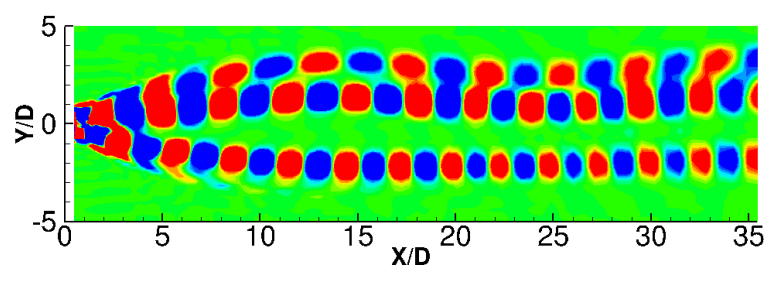

(d)

$$
\omega_{z}:-1.0 \mathrm{E}+00 \quad-5.0 \mathrm{E}-01 \quad 0.0 \mathrm{E}+00 \quad 5.0 \mathrm{E}-01 \quad 1.0 \mathrm{E}+00
$$

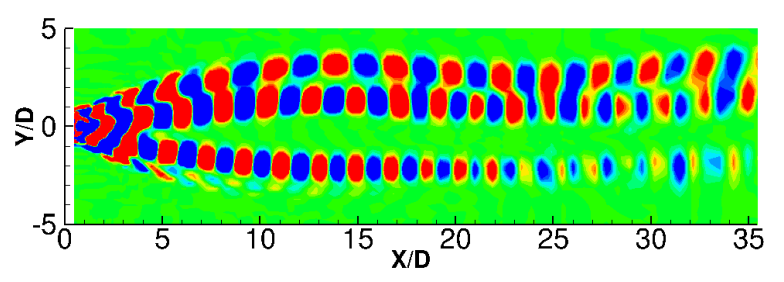

(f)

$$
\omega_{z}:-1.0 \mathrm{E}+00 \quad-5.0 \mathrm{E}-01 \quad 0.0 \mathrm{E}+00 \quad 5.0 \mathrm{E}-01 \quad 1.0 \mathrm{E}+00
$$

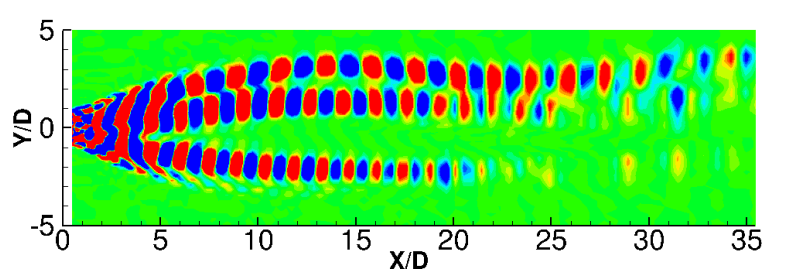

(h)

Figure 25. Spatial patterns of the POD modes for case $R e=400$ and $S t=0.054$ : (a) $\operatorname{mode} 1$; (b) mode 2 ; (c) mode 3 ; (d) mode 4 ; (e) mode 5 ; (f) mode 6 ; (g) mode 7 ; (h) mode 8. 


\section{References}

${ }^{1}$ Singh, A. P., De, A. K., Carpenter, V. K., Eswaran, V., and Muralidhar, K., "Flow past a Transversely Oscillating Square Cylinder in Free Stream at Low Reynolds Numbers", International Journal for Numerical Methods in Fluids, Vol. 61, No. 6, 2009, pp. 658-682.

${ }^{2}$ Yang, S.-J., Chang, T.-R., and Fu, W.-S., "Numerical simulation of flow structures around an oscillating rectangular cylinder in a channel flow", Computational Mechanics, Vol. 35, No. 5, pp. 342-351, 2005.

${ }^{3}$ Sen, S., and Mittal, S., "Free vibration of a square cylinder at low Reynolds numbers", Journal of Fluids and Structures, Vol. 27, No. 5-6, pp. 875-884, 2011.

${ }^{4}$ Luo, S. C., "Vortex wake of a transversely oscillating square cylinder: A flow visualization analysis", Journal of Wind Engineering and Industrial Aerodynamics, Vol. 45, No. 1, pp. 97-119, 1992.

${ }^{5}$ Huynh, H. T., "A Flux Reconstruction Approach to High-Order Schemes including Discontinuous Galerkin Methods", AIAA Paper, 4079, 2007.

${ }^{6}$ Wang, Z. J., and Gao, H. Y., "A Unifying Lifting Collocation Penalty Formulation Including the Discontinuous Galerkin, Spectral Volume/Difference Methods for Conservation Laws on Mixed Grids", Journal of Computational Physics, vol. 228, issue 21, pp. 8161-8186, 2009.

${ }^{7}$ Vincent, P. E., Castonguay, P., and Jameson, A., "A new class of high-order energy stable flux reconstruction schemes", J. Sci. Comput., vol. 47, issue 1, pp. 50-72, 2011.

${ }^{8}$ Huynh, H. T., Wang, Z. J., and Vincent, P. E., "High-order methods for computational fluid dynamics: A brief review of compact differential formulations on unstructured grids", Computers \& Fluids, vol. 98, pp. 209-220, 2014.

${ }^{9}$ Wang, Z. J., and Huynh, H. T., "A review of flux reconstruction or correction procedure via reconstruction method for the Navier-Stokes equations", Mechanical Engineering Reviews, vol. 3, pp. 1-16, 2016.

${ }^{10} \mathrm{Yu}$, M. L., Wang, Z. J., and Liu, Y., "On the accuracy and efficiency of discontinuous Galerkin, spectral difference and correction procedure via reconstruction methods", Journal of Computational Physics, vol. 259, pp. 70-95, 2014.

${ }^{11}$ Wang, L., and Yu, M. L., "A High-Order Dual-Time Stepping FR/CPR Method for Unsteady Incompressible Navier-Stokes Equations on Unstructured Moving Grids", 2016 AIAA Science and Technology Forum and Exposition, 4 - 8 January 2016, San Diego, California.

${ }^{12} \mathrm{Yu}$, M. L., and Wang, L., "A High-Order Flux Reconstruction/Correction Procedure via Reconstruction Formulation for Unsteady Incompressible Flow on Unstructured Moving Grids", in press, Computers \& Fluids, 2016.

${ }^{13}$ Yu, M. L., Wang, Z.J., Hu, H., "A High-Order Spectral Difference Method for Unstructured Dynamic Grids", Computers \& Fluids, vol. 48, issue 1, pp. 84-97, 2011.

${ }^{14}$ Rowley, C. W., Mezić, I., Bagheri, S., Schlatter, P., and Henningson, D. S., "Spectral analysis of nonlinear flows", Journal of Fluid Mechanics, Vol. 641, pp. 115-127, 2009.

${ }^{15}$ Schmid, P. J., "Dynamic Mode Decomposition of Numerical and Experimental Data", Journal of Fluid Mechanics, Vol. 656, 2010, pp. 5-28.

${ }^{16}$ Schmid, P. J., "Application of the Dynamic Mode Decomposition to Experimental Data", Experiments in Fluids, Vol. 50, No. 4, 2011, pp. 1123-1130.

${ }^{17}$ Schmid, P. J., Li, L., Juniper, M. P., and Pust, O., "Applications of the Dynamic Mode Decomposition", Theoretical and Computational Fluid Dynamics, Vol. 25, Nos. 1-4, 2011, pp. 249-259.

${ }^{18}$ Jovanović, M. R., Schimid, P. J., and Nichols, J. W., "Sparsity-Promoting Dynamic Mode Decomposition", Physics of Fluids, Vol. 26, No. 2, 2014, pp. 024103.

${ }^{19} \mathrm{Tu}$, J. H., Rowley, C. W., Luchtenburg, D. M., Brunton, S. L., and Kutz, J. N., "On Dynamic Mode Decomposition: Theory and Applications", Journal of Computational Dynamics, Vol. 1, No. 2, 2014, pp. 391-421.

${ }^{20}$ Mariappan, S., Gardner, A. D., Richter, K., and Raffel, M., "Analysis of Dynamic Stall Using Dynamic Mode Decomposition Technique", AIAA Journal, 2014 52:11, 2427-2439

${ }^{21}$ Zhang, Q., Liu, Y., and Wang, S., "The identification of coherent structures using proper orthogonal decomposition and dynamic mode decomposition", Journal of Fluids and Structures, Vol. 49, pp. 53-72, 2014.

${ }^{22}$ Horchler, T., Mani, K. V., and Hannemann, K., "Dynamic Mode Decomposition of Backward Facing Step Flow Simulation Data", AIAA 2015-3411, AIAA Aviation, 22-26 June 2015, Dallas, TX, 22nd AIAA Computational Fluid Dynamics Conference.

${ }^{23}$ Wang, Z. J., " Adaptive high-order methods in computational fluid dynamics", World Scientific, Advances in computational fluid dynamics, Vol. 2., 2011.

${ }^{24}$ Roe, P. L., "Approximate Riemann solvers, parameter vectors and difference schemes", J. Comput. Phys. , 43 (2), pp. 357-372, 1981.

${ }^{25}$ Bassi, F., and Rebay, S., "Discontinuous Galerkin solution of the Reynolds-averaged Navier-Stokes and $k-\omega$ turbulence model equations", Computers \& Fluids , 34, 507-540, 2005. 
${ }^{26}$ Persson, P.-O., and Peraire, J.. "Newton-GMRES preconditioning for discontinuous Galerkin discretizations of the Navier-Stokes equations", SIAM J. Sci. Comput. , 30, pp. 2709-2733, 2008.

${ }^{27} \mathrm{Yu}$, M. L., Hu, H., and Wang, Z. J.. "Experimental and numerical investigations on the asymmetric wake vortex structures of an oscillating airfoil", AIAA-2012-0299, the 50th Aerospace Sciences Meeting Including the New Horizons Forum and Aerospace Exposition, Nashville, Tennessee, Jan. 9-12, 2012.

${ }^{28}$ Noack, B. R., Afanasiev, K., Morzynski, Tadmor, G., and Thiele,F., "A hierarchy of low-dimensional models for the transient and post-transient cylinder wake", Journal of Fluid Mechanics, 497, pp. 335-363, 2003.

${ }^{29}$ Lumley, J. L., "Stochastic tools in turbulence." Academic Press, 1970.

${ }^{30}$ Sirovich, L., "Turbulence and the dynamics of coherent structures part I: coherent structures.", Quarterly of Applied Mathematics 45, pp. 561-571,1987.

${ }^{31}$ Sayadi, T., Nichols, J. W., Schmid, P. J., and Jovanović, M. R., "Dynamic mode decomposition of H-type transition to turbulence." Center for Turbulence Research, Proceedings of the Summer Program 2012. 